\title{
Finite element formulation for the analysis of multilayered beams based on the principle of stationary complementary strain energy
}

\author{
Phe Van Pham ${ }^{\mathrm{a}, 1}$, Magdi Mohareb ${ }^{\mathrm{a}}$, Amir Fam $^{\mathrm{b}}$ \\ ${ }^{a}$ Department of Civil Engineering, University of Ottawa, Ottawa, ON, Canada K1N 6N5 \\ ${ }^{\mathrm{b}}$ Department of Civil Engineering, Queen's University, Kingston, ON, Canada K7L 3N6
}

\begin{abstract}
A family of finite elements for the analysis of orthotropic multilayered beams with monosymmetric cross-sections is developed based on the principle of stationary complementary energy. The longitudinal normal stress field is postulated as polynomial and Heaviside step function series and substituted into the infinitesimal equilibrium conditions to develop expressions for the shear and transverse stress fields. The statically admissible stress fields thus derived are then adopted within the complementary energy variational principle framework to develop a family of finite elements. The distinguishing features of the solution are: (i) it captures the nonlinear distribution of the stress fields along the section depth and steep stress gradients typically occurring near bondline ends of multilayer beams, (ii) unlike conventional solutions based on the principle of stationary potential energy which predict jumps in the shear and peeling stresses at interfaces of adjacent layers, the present solution satisfies equilibrium in an exact infinitesimal sense at layer interfaces and thus ensures continuity of the stress fields across the interface, (iii) it naturally captures the effects of transverse shear and transverse normal stresses, and (iv) it consistently converges to the displacements from above, in contrast to conventional finite element solutions where convergence is typically from below. The versatility of the solution is then illustrated in applications involving wood beams and steel beams strengthened with GFRP plates and sandwich beams with soft cores.
\end{abstract}

Keywords: High order beam theory, complementary strain energy, variational principle, finite element, orthotropic materials, layered beams, sandwich beams, GFRP, beam strengthening.

This article is to be cited as: Pham PV, Mohareb M, Fam A, 2018, Finite element formulation for the analysis of multilayered beams based on the principle of stationary complementary strain energy, Engineering Structures, 167: 287-307, ISSN 0141-0296. A copy-edited version of this article can be obtained at: $\underline{\text { https://doi.org/10.1016/j.engstruct.2018.04.014 }}$

\footnotetext{
${ }^{1}$ Corresponding author. Tel: (+1) 6135625800 ext. 6066; Email: ppham040@uottawa.ca (P.Pham).
} 


\section{Motivation and Literature review}

The adoption of conventional beam theories (e.g., the Euler-Bernouilli or Timoshenko theories) in the analysis of multilayered beams is associated with inconsistencies and limitations. The Euler-Bernoulli beam kinematic assumption postulating that plane section remains plane and normal to the beam axis throughout deformation, implies the neglect of shear deformation effects and thus tends to grossly over-predict the stiffness of short-span composite beams. An improved solution accounting for shear deformation is the Timoshenko beam assumption which retains the plane section assumption but relaxes the normality condition between the cross-section plane and the beam axis. The Timoshenko beam kinematics however introduce non-zero shear strains (and hence stresses) at the extreme fibers of the cross-section and thus violate the traction boundary conditions at extreme fibers where externally applied shear stresses typically vanish. To remedy such contradictions, various researchers have formulated correction shear coefficients (e.g., [1, 5]) that depend on whether the analysis is static or dynamic (e.g., [6,9]) and are section dependent. Higher order beam theories with improved kinematic assumptions include the work of Stephen and Levinson [10], Reddy [11], Heyliger and Reddy [9], Shu and Sun [12], Jha et al [13], Kant and Manjunatha [14], Zhang et al [15] where the longitudinal displacement field is assumed as a third order polynomial. Such studies are however limited to rectangular cross-sections ([16,17]). Another class of solutions based on assumed higher order polynomials for the longitudinal displacements were developed by Carrera and Giunta [18], Carrera et al. [19], and Groh and Weaver [20]. Such theories have the advantage of capturing shear deformation effects without the need of introducing shear modification factors. Some of these theories $([18,19])$ have additionally captured the effect of transverse normal stresses.

Irrespective of the kinematic assumptions made in the above studies, when used in conjunction with the principle of minimum potential energy (referred to as the conventional treatment subsequently), they lead to approximate differential equations of equilibrium providing a stiffer representation of the structure compared to that based on the exact infinitesimal equilibrium conditions (e.g., solutions based on the theory of elasticity). Another characteristic observed when applying the conventional treatment to composite beams with layers of different materials is that the postulation of continuous displacement fields (either linear, cubic, or higher order), while 
ensuring continuity of strains at the interfaces of adjoining materials, lead to discontinuous stresses at the interfaces when such strains are multiplied by the different constitutive properties of the interfaces of adjoining materials. Such stress discontinuities violate the local shear and transverse equilibrium conditions at the interface. This disadvantage is observed not only in analytical solutions but also in finite element solutions based on the principle of stationary potential energy. In 3D finite element solutions, such discrepancies can be minimized, but not eliminated, by adopting a fine mesh in the transverse direction, albeit such a measure results in an undesirable computational expense. Within this context, the present study contributes to remedy the limitations of the conventional treatment by adopting the principle of complementary potential energy in conjunction with statically admissible high-order stress fields to develop an improved finite element solution that satisfies continuity of shear and transverse stress fields at layer interfaces.

A comparison between the conventional treatment and the complementary energy solution is depicted in Figure 1. Under the conventional stationary potential energy principle, displacement fields are initially postulated, hence satisfying compatibility of strains in an exact pointwise sense. The stationarity condition of the total potential energy functional is then evoked to yield approximate equilibrium equations which tend to overestimate the stiffness of the structure. In contrast, under the principle of stationary total complementary strain energy, stress fields exactly satisfying the equilibrium conditions in an exact pointwise sense are postulated and the complementary strain energy principle is then expressed in terms of the unknown stress fields. The condition of stationarity of the complementary strain energy functional then yields approximate compatibility equations in terms of the stress fields, which lead to a solution that tends to underestimate the stiffness of the structure. Recently, Groh and Tessler [21] developed a refined zigzag theory for the analysis for multi-layered beam with doubly symmetric crosssections based on the Reissner variational principle.

A comparatively limited number of finite element solutions have adopted the principle of stationary complementary energy. Wunderlich and Pilkey [22] developed complementary strain energy solutions for plane stress problems by postulating a longitudinal stress field that varies linearly across the section height. Erkmen and Mohareb [23] derived a complementary energy solution for the torsional analysis of thin-walled beams. Erkmen and Mohareb [24] developed a complementary energy variational principle for the buckling analysis of thin-walled open members based on the principle of stationary complementary energy. Zhao et al. [25] adopted a 
two-dimensional elasticity theory solution in conjunction with the principle of stationary complementary energy for the analysis of adhesive-bonded single-lap joints. By adopting longitudinal normal stress fields that linearly vary in the transverse direction, and incorporating shear stresses, $\mathrm{Wu}$ and Jensen [16] developed a solution for layered beams to predict interfacial shear and normal stresses. However, discrepancies between their solutions and two-dimensional finite element analyses under ANSYS were observed near the bond ends and were attributed to the postulated approximate linear distribution for longitudinal normal stresses.

Within the above context, the present study develops a higher order beam theory that naturally incorporates shear and normal transverse stresses. Since the theory aims at the accurate predictions of interfacial stresses, the longitudinal normal stresses are assumed to have a general non-linear distribution along the section height. In order to adopt the jumps of the longitudinal stresses at layer interfaces, Heaviside step functions are introduced in the characterization of the longitudinal stress field.

Conventional Treatment

Assume kinematically admissible strains typically by assuming displacement functions

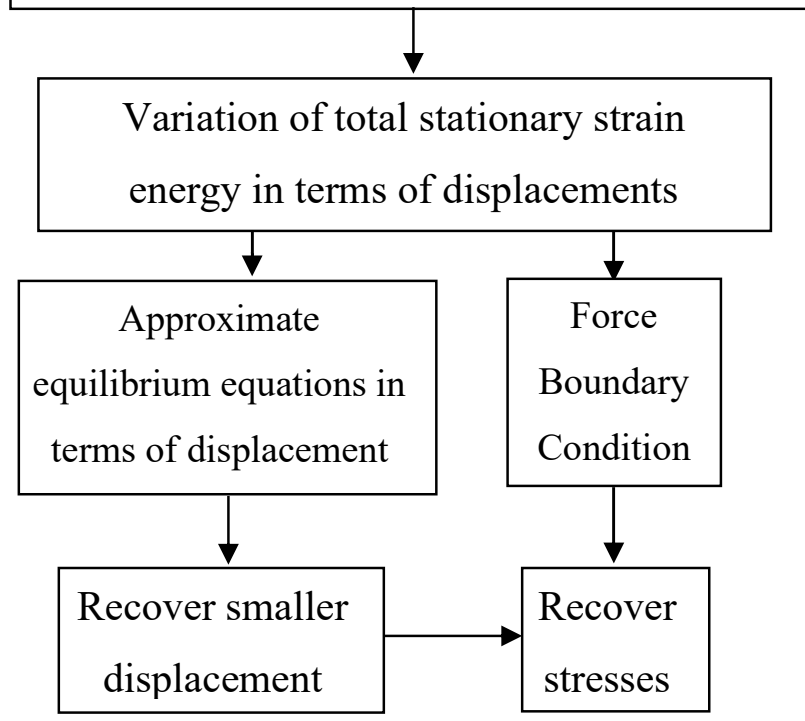

Present Study

Assume statically admissible stresses Exactly satisfying equilibrium conditions

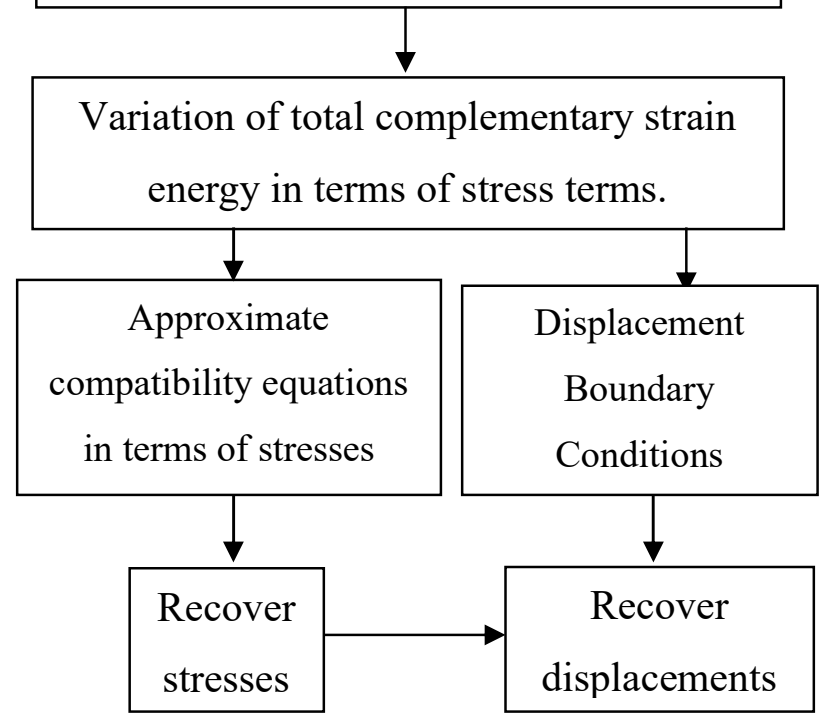

Figure 1. Conventional solutions versus present complementary energy solution

\section{Statement of the Problem}

A prismatic multi-layer beam with an arbitrary mono-symmetric cross-section is subjected to general body forces $p_{y}(y, z)$ and $p_{z}(y, z)$ in the transverse and longitudinal directions. Externally 
applied surface tractions $\sigma\left(-h_{1}, z\right), \tau\left(-h_{1}, z\right), \sigma\left(h_{2}, z\right), \tau\left(h_{2}, z\right)$ are applied to the top and bottom faces (Fig.2a). It is required to formulate a finite element formulation for the problem. The coordinate system $O X Y Z$ is adopted, in which origin $O$ is an arbitrary point on the cross-section (Figure 2a and b). Cross-sectional dimension notations are depicted on Figure $2 \mathrm{~b}$ in which $h_{1}$ and $h_{2}$ respectively denote the distances from top and bottom fibers and $b(y)$ is the cross-section width as a function of the coordinate $y\left(-h_{1} \leq y \leq h_{2}\right)$.

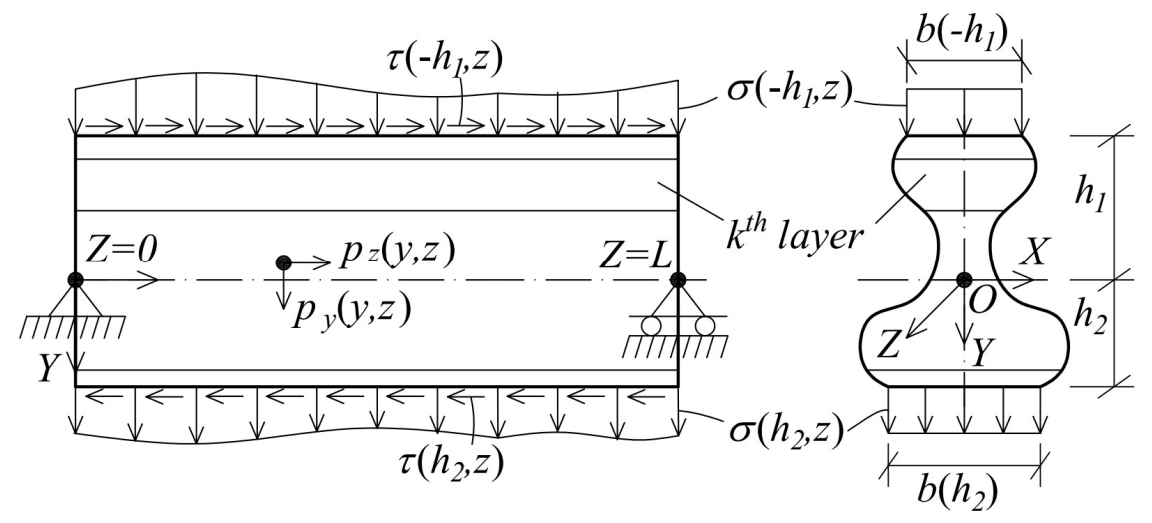

(a)

(b)

Figure 2. Tractions and body forces applied to the beam (a) Profile view and (b) Cross-section view

\section{Assumptions}

The following assumptions are made:

a) The beam is prismatic and subjected to longitudinal and transverse loads,

b) The beam consists of $p$ layers with $p-1$ interfaces.

c) All materials are linearly elastic orthotropic,

d) Perfect bonding is assumed between the various layers,

e) The longitudinal normal stresses take the form $\sigma_{z}(y, z)=\sum_{i=1}^{n} y^{i-1} F_{i}(z)+\sum_{k=1}^{p-1} H\left(y-y_{k}\right) J_{k}(z)$ where $F_{i}(z)$ are unknown functions of longitudinal coordinate $z, y$ is the transverse coordinate and $n$ is a positive integer. The second summation is on the number of interfaces $k=1,2, \ldots, p-1$ and $J_{k}(z)$ are unknown functions. The term $H\left(y-y_{k}\right)$ is the Heaviside step function defined as $H\left(y-y_{k}\right)=0$ when $y<y_{k}$ and $H\left(y-y_{k}\right)=1.0$ when $y>y_{k}$. While the Heaviside step function is discontinuous at the interface $y=y_{k}$ (and hence accurately 
represents the stress jumps expected at the layer interfaces), its first integral $\int_{-h_{1}}^{y} H\left(y-y_{k}\right) d y$ and second integral $\int_{-h_{1}}^{y} \int_{-h_{1}}^{y} H\left(y-y_{k}\right) d y d y$ are continuous functions at interfaces $y=y_{k}$, and thus emulate the expected continuous shear and transverse stress fields across the interfaces. Also, the transverse normal stresses $\sigma_{y}(y, z)$ and shear stresses $\tau(y, z)$ (Figure 3) are to be determined from infinitesimal equilibrium conditions. Other out-of-plane stress components are assumed negligible.

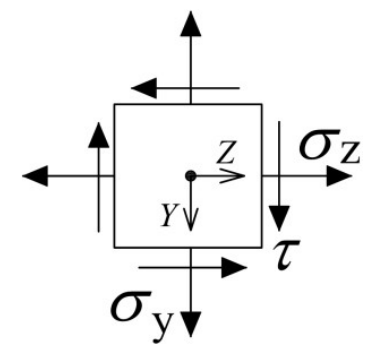

Figure 3. Stresses components considered in the present theory

\section{Formulation}

\subsection{Statically Admissible Stress Fields}

As discussed in the assumption, the longitudinal stresses $\sigma_{z}(y, z)$ are postulated to take the form

$$
\sigma_{z}(y, z)=\sum_{i=1}^{n} y^{i-1} F_{i}(z)+\sum_{k=1}^{p-1} H\left(y-y_{k}\right) J_{k}(z)
$$

For the stress fields to be statically admissible, the stress fields $\sigma_{y}(y, z)$ and $\tau(y, z)$ (Figure 4) need to satisfy the equilibrium conditions in an exact pointwise sense. The equilibrium conditions take the form

$$
\begin{aligned}
& \frac{\partial}{\partial y}\left[b(y) \sigma_{y}(y, z)\right]+\frac{\partial}{\partial z}[b(y) \tau(y, z)]=b(y) p_{y}(y, z) \\
& \frac{\partial}{\partial y}[b(y) \tau(y, z)]+\frac{\partial}{\partial z}\left[b(y) \sigma_{z}(y, z)\right]=b(y) p_{z}(y, z)
\end{aligned}
$$

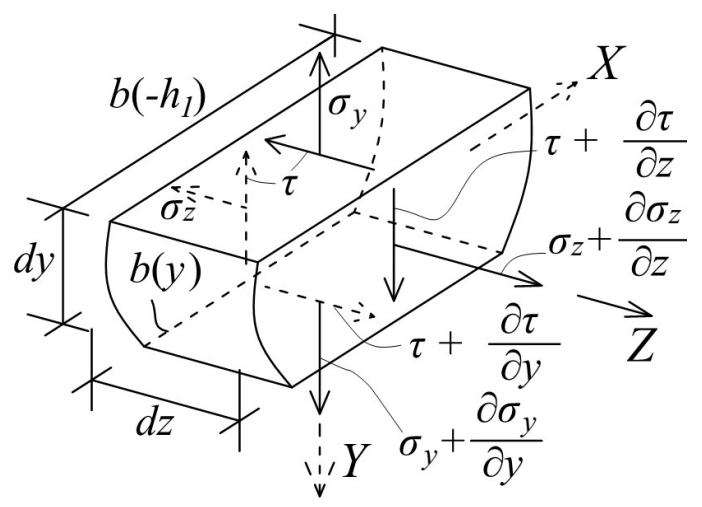




\section{Figure 4. Infinitesimal element in equilibrium}

From Eq.(1), by substituting into Eq. (2b), and integrating both sides with respect to $y$ from the top fiber at height $-h_{1}$ to an arbitrary height $y$, one recovers the expression of shear stresses $\tau(y, z)$. Next, given the expression for $\tau(y, z)$, by substituting into Eq. (2a) and integrating with

respect to $y$ from $-h_{1}$ to $y$, one recovers an expression for the transverse normal stress $\sigma_{y}(y, z)$ . The resulting stress fields take the form

$$
\begin{aligned}
\tau(y, z) & =\bar{\tau}(y, z)-\sum_{i=1}^{n} \alpha_{i}(y) F_{i}{ }^{\prime}(z)-\sum_{k=1}^{p-1} a_{k}(y) J_{k}{ }^{\prime}(z) \\
\sigma_{y}(y, z) & =\bar{\sigma}_{y}(y, z)+\sum_{i=1}^{n} \beta_{i}(y) F_{i}^{\prime \prime}(z)+\sum_{k=1}^{p-1} c_{k}(y) J_{k}{ }^{\prime \prime}(z)
\end{aligned}
$$

in which functions

$$
\begin{aligned}
\bar{\tau}(y, z) & =\frac{1}{b(y)} b\left(-h_{1}\right) \tau\left(-h_{1}, z\right)+\frac{1}{b(y)} \int_{-h_{1}}^{y} b(\xi) p_{z}(\xi, z) d \xi \\
\bar{\sigma}_{y}(y, z) & =\frac{1}{b(y)} b\left(-h_{1}\right) \sigma_{y}\left(-h_{1}, z\right)+\frac{1}{b(y)} \int_{-h_{1}}^{y} b(\xi) p_{y}(\xi, z) d \xi-\frac{1}{b(y)} b\left(-h_{1}\right) \frac{\partial \tau\left(-h_{1}, z\right)}{\partial z} \\
& -\frac{1}{b(y)} \int_{-h_{1}-h_{1}}^{y} \int^{\eta} b(\xi) \frac{\partial p_{z}(\xi, z)}{\partial z} d \xi d \eta
\end{aligned}
$$

depend on surface tractions $\tau\left(-h_{1}, z\right)$ and $\sigma_{y}\left(-h_{1}, z\right)$, and body forces $p_{z}(y, z)$ and $p_{y}(y, z)$. Also, in Eq. (3a-b), $\alpha_{i}(y), \beta_{i}(y), a_{k}(y)$ and $c_{k}(y)$ are distribution functions depending on the section geometry and defined as

$$
\begin{aligned}
& \alpha_{i}(y)=\frac{1}{b(y)} \int_{-h_{1}}^{y} b(\xi) \xi^{i-1} d \xi ; \quad \beta_{i}(y)=\frac{1}{b(y)} \int_{-h_{1}-h_{1}}^{y} \int^{\eta} b(\xi) \xi^{i-1} d \xi d \eta \\
& a_{k}(y)=\frac{1}{b(y)} \int_{-h_{1}}^{y} b(y) H\left(y-y_{k}\right) d y ; \quad c_{k}(y)=\frac{1}{b(y)} \int_{-h_{1}-h_{1}}^{y} \int^{y} b(y) H\left(y-y_{k}\right) d y d y
\end{aligned}
$$

Equations (1) and (3a-b) satisfy the traction boundary condition at the top face, but not those at bottom face $y=h_{2}$. By equating the stresses in Eqs. (3a-b) to the specified traction $\tau\left(h_{2}, z\right)$ and $\sigma_{y}\left(h_{2}, z\right)$, functions $F_{n-1}(z)$ and $F_{n}(z)$ can be related to the remaining functions $F_{i}(z)$ where $i=1, \ldots, n-2$ (Appendix A). From Equations A.5 and A.13a-d, by substituting into Eq. (1) and (3a-b), one obtains 


$$
\begin{aligned}
\sigma_{z}(y, z) & =\mathbf{a}_{\mathbf{1}}(y)^{T} \mathbf{F}(z)+\mathbf{a}_{\mathbf{2}}(y)^{T} \mathbf{F}(0)+\mathbf{a}_{\mathbf{3}}(y, z)^{T} \mathbf{F}^{\prime}(0)+\mathbf{b}_{\mathbf{1}}(y, z)^{T} \mathbf{R}(0)+\sigma_{z}^{*}(y, z) ; \\
\tau(y, z) & =\mathbf{a}_{\mathbf{4}}(y)^{T} \mathbf{F}^{\prime}(z)+\mathbf{a}_{\mathbf{5}}(y)^{T} \mathbf{F}^{\prime}(0)+\mathbf{b}_{\mathbf{2}}(y)^{T} \mathbf{R}(0)+\tau^{*}(y, z) ; \\
\sigma_{y}(y, z) & =\mathbf{a}_{\mathbf{6}}(y)^{T} \mathbf{F}^{\prime \prime}(z)+\sigma_{y}^{*}(y, z)
\end{aligned}
$$

where functions $F_{i}(z), J_{p}(z)$ have been grouped into the following vectors:

$$
\begin{array}{lllllllll}
\mathbf{F}(z)_{1 \times(m+p-1)}^{T}=\left\langle F_{1}(z)\right. & F_{1}(z) & \ldots & F_{i}(z) \ldots & F_{m}(z) \mid J_{1}(z) & J_{2}(z) & \ldots & J_{k}(z) \ldots & \left.J_{p-1}(z)\right\rangle ; \\
\mathbf{R}(0)_{1 \times 3}^{T}=\langle N(0) & Q(0) & M(0)\rangle ; & (m=n-2)
\end{array}
$$

in which stress resultants $N(0), Q(0), M(0)$ (Fig.5a) are defined as

$$
N(0)=\int_{A} \sigma_{z}(y, 0) d A ; \quad Q(0)=\int_{A} \tau(y, 0) d A ; \quad M(0)=\int_{A} y \sigma_{z}(y, 0) d A
$$

and $A$ is the cross-sectional area. The contributions of applied loads are defined as

$$
\begin{aligned}
\sigma_{z}^{*}(y, z) & =\int_{0}^{z} \int_{0}^{z}\left[y^{n-2} \rho_{1}(z)+y^{n-1} \rho_{2}(z)\right] d z d z+z\left(y^{n-2} \varsigma_{11}+y^{n-1} \varsigma_{21}\right) \\
\tau^{*}(y, z) & =\bar{\tau}(y, z)-\int_{0}^{z}\left[\alpha_{n-1}(y) \rho_{1}(z)+\alpha_{n}(y) \rho_{2}(z)\right] d z-\alpha_{n-1}(y) \varsigma_{11}-\alpha_{n}(y) \varsigma_{21} \\
\sigma_{y}^{*}(y, z) & =\bar{\sigma}_{y}(y, z)+\beta_{n-1}(y) \rho_{1}(z)+\beta_{n}(y) \rho_{2}(z)
\end{aligned}
$$

where $\rho_{1}(z), \rho_{2}(z), \varsigma_{11}, \varsigma_{21}$ are defined in Appendices A. In Eq. (6), the following vectors of crosssectional parameters have been introduced

$$
\begin{aligned}
& \mathbf{a}_{\mathbf{1}}(y)^{T}=\left\langle a_{11}(y) \quad a_{12}(y) \quad \ldots a_{1 i}(y) \ldots \quad a_{1 m}(y) \mid g_{11}(y) \quad g_{12}(y) \quad \ldots g_{1 k}(y) \ldots \quad g_{1 p-1}(y)\right\rangle, \\
& \mathbf{a}_{2}(y)^{T}=\left\langle a_{21}(y) \quad a_{22}(y) \quad \ldots a_{2 i}(y) \ldots \quad a_{2 m}(y) \mid g_{21}(y) \quad g_{22}(y) \quad \ldots g_{2 k}(y) \ldots \quad g_{2 p-1}(y)\right\rangle, \\
& \mathbf{a}_{3}(y, z)^{T}=z\left\langle a_{31}(y) \quad a_{32}(y) \quad \ldots a_{3 i}(y) \ldots \quad a_{3 m}(y) \mid g_{31}(y) \quad g_{32}(y) \quad \ldots g_{3 k}(y) \ldots \quad g_{3 p-1}(y)\right\rangle,
\end{aligned}
$$

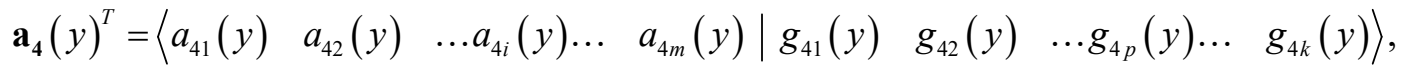

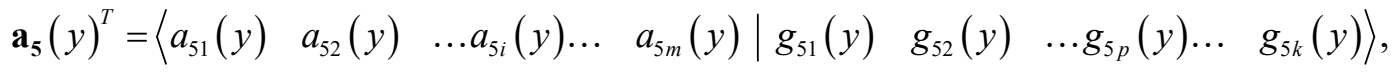

$$
\begin{aligned}
& \mathbf{a}_{6}(y)^{T}=\left\langle a_{61}(y) \quad a_{62}(y) \quad \ldots a_{6 i}(y) \ldots \quad a_{6 m}(y) \mid g_{61}(y) \quad g_{62}(y) \quad \ldots g_{6 p}(y) \ldots \quad g_{6 k}(y)\right\rangle, \\
& \mathbf{b}_{1}(y, z)^{T}=\left\langle b_{11}(y) \quad z b_{12}(y) \quad b_{13}(y)\right\rangle, \quad \mathbf{b}_{2}(y)=\left\langle\begin{array}{lll}
0 & b_{22}(y) & 0
\end{array}\right\rangle, \\
& \text { in which } \\
& \begin{array}{llrl}
a_{1 i}(y)=y^{i-1}-y^{n-2} \lambda_{1 i}-y^{n-1} \lambda_{2 i} ; & g_{1 k}(y)=H\left(y-y_{k}\right)-y^{n-2} \xi_{1 k}-y^{n-1} \xi_{2 k} \\
a_{2 i}(y)=y^{n-2} \varsigma_{33 i}+y^{n-1} \varsigma_{43 i}+y^{n-2} \lambda_{1 i}+y^{n-1} \lambda_{2 i} ; & g_{2 k}(y)=y^{n-2} \varsigma_{34 k}+y^{n-1} \varsigma_{44 k}+y^{n-2} \xi_{1 k}+y^{n-1} \xi_{2 k} \\
a_{3 i}(y)=y^{n-2} \varsigma_{13 i}+y^{n-1} \varsigma_{23 i}+y^{n-2} \lambda_{1 i}+y^{n-1} \lambda_{2 i} ; & g_{3 k}(y)=y^{n-2} \varsigma_{14 k}+y^{n-1} \varsigma_{24 k}+y^{n-2} \xi_{1 k}+y^{n-1} \xi_{2 k} \\
a_{4 i}(y)=\alpha_{n-1}(y) \lambda_{1 i}+\alpha_{n}(y) \lambda_{2 i}-\alpha_{i}(y) ; & g_{4 k}(y)=\alpha_{n-1}(y) \xi_{1 k}+\alpha_{n}(y) \xi_{2 k}-a_{k}(y) \\
a_{5 i}(y)=-\alpha_{n-1}(y) \varsigma_{13 i}-\alpha_{n}(y) \varsigma_{23 i}-\alpha_{n-1}(y) \lambda_{1 i}-\alpha_{n}(y) \lambda_{2 i} ; \\
g_{5 k}(y)=-\alpha_{n-1}(y) \varsigma_{14 k}-\alpha_{n}(y) \varsigma_{24 k}-\alpha_{n-1}(y) \xi_{1 k}-\alpha_{n}(y) \xi_{2 k} ; \\
a_{6 i}(y)=\beta_{i}(y)-\beta_{n-1}(y) \lambda_{1 i}-\beta_{n}(y) \lambda_{2 i} ; & g_{6 k}(y)=c_{k}(y)-\beta_{n-1}(y) \xi_{1 k}-\beta_{n}(y) \xi_{2 k} \\
b_{11}(y)=y^{n-2} \varsigma_{31}+y^{n-1} \varsigma_{41}, & b_{12}(y)=y^{n-2} \varsigma_{12}+y^{n-1} \varsigma_{22}, \\
b_{13}(y)=y^{n-2} \varsigma_{32}+y^{n-1} \varsigma_{42}, & b_{22}(y)=-\left[\alpha_{n-1}(y) \varsigma_{12}+\alpha_{n}(y) \varsigma_{22}\right]
\end{array}
\end{aligned}
$$


Constants $\lambda_{1 i}, \lambda_{2 i}, \xi_{1 k}, \xi_{2 k}$, have been defined in Appendix A while constants $\varsigma_{12}, \varsigma_{22}, \varsigma_{31}, \varsigma_{32}, \varsigma_{41}$, $\varsigma_{42}, \varsigma_{13 i}, \varsigma_{23 i}, \varsigma_{33 i}, \varsigma_{43 i}, \varsigma_{14 k}, \varsigma_{24 k}, \varsigma_{34 k}, \varsigma_{44 k}$ have been defined in Appendix B.

\subsection{Interpolation of stress fields}

Functions $F_{i}(z), i=1,2, \ldots, m$ and $J_{k}, k=1,2, \ldots, p-1$ are assumed to follow a cubic distribution in coordinate $z$. One can express each stress function $F_{i}(z), J_{k}(z)$ in terms of the nodal values as

$$
F_{i}(z)=\overline{\mathbf{S}}_{1 \times 2}^{T}\left\{\overline{\mathbf{F}}_{\mathbf{N} i}\right\}_{2 \times 1}+\overline{\overline{\mathbf{S}}}_{1 \times 2}^{T}\left\{\overline{\overline{\mathbf{F}}}_{\mathbf{N} i}\right\}_{2 \times 1} ; \quad J_{i}(z)=\overline{\mathbf{S}}_{1 \times 2}^{T}\left\{\overline{\mathbf{J}}_{\mathbf{N} i}\right\}_{2 \times 1}+\overline{\overline{\mathbf{S}}}_{1 \times 2}^{T}\left\{\overline{\overline{\mathbf{J}}}_{\mathbf{N} i}\right\}_{2 \times 1}
$$

in which $\overline{\mathbf{S}}_{1 \times 2}^{T}, \overline{\overline{\mathbf{S}}}_{1 \times 2}^{T}$ are the vectors of interpolation functions defined as

$$
\overline{\mathbf{S}}_{1 \times 2}^{T}=\frac{1}{L^{3}}\left\langle 2 z^{3}-3 z^{2} L+L^{3}: z^{3} L-2 z^{2} L^{2}+z L^{3}\right\rangle ; \quad \overline{\mathbf{S}}_{1 \times 2}^{T}=\frac{1}{L^{3}}\left\langle-2 z^{3}+3 z^{2} L: z^{3} L-z^{2} L^{2}\right\rangle
$$

and

$$
\begin{aligned}
& \left\langle\overline{\mathbf{F}}_{\mathbf{N} i}\right\rangle_{1 \times 2}^{T}=\left\langle F_{i}(0): F_{i}^{\prime}(0)\right\rangle ; \quad\left\langle\overline{\overline{\mathbf{F}}}_{\mathbf{N} i}\right\rangle_{1 \times 2}^{T}=\left\langle F_{i}(L) F_{i}^{\prime}(L)\right\rangle ; \\
& \left\langle\overline{\mathbf{J}}_{\mathbf{N} i}\right\rangle_{1 \times 2}^{T}=\left\langle J_{i}(0): J_{i}^{\prime}(0)\right\rangle ; \quad\left\langle\overline{\overline{\mathbf{J}}}_{\mathbf{N} i}\right\rangle_{1 \times 2}^{T}=\left\langle J_{i}(L): J_{i}^{\prime}(L)\right\rangle ;
\end{aligned}
$$

are the vectors of nodal forces. It is convenient to express the nodal force functions $\{\mathbf{F}(z)\}$ as

$$
\{\mathbf{F}(z)\}_{(m+p-1) \times 1}=\left[\boldsymbol{\Lambda}_{\mathbf{F}}(z)\right]\{\overline{\mathbf{P}}\}
$$

in which the interpolation function matrix $\left[\boldsymbol{\Lambda}_{\mathbf{F}}(\mathbf{z})\right]$ is defined as

$$
\boldsymbol{\Lambda}_{\mathbf{F}}(z)_{(m+p-1) \times(4 m+4 p-4+3)}=\left[\begin{array}{cccc:cc:ccc}
\overline{\mathbf{S}}_{1 \times 2}^{T} & \mathbf{0}_{1 \times 2}^{T} & \ldots & \mathbf{0}_{1 \times 2}^{T} & \mathbf{0}_{1 \times 3}^{T} & \overline{\mathbf{S}}_{1 \times 2}^{T} & \mathbf{0}_{1 \times 2}^{T} & \ldots & \mathbf{0}_{1 \times 2}^{T} \\
\mathbf{0}_{1 \times 2}^{T} & \overline{\mathbf{S}}_{1 \times 2}^{T} & \ldots & \mathbf{0}_{1 \times 2}^{T} & \mathbf{0}_{1 \times 3}^{T} & \mathbf{0}_{1 \times 2}^{T} & \overline{\mathbf{S}}_{1 \times 2}^{T} & \ldots & \mathbf{0}_{1 \times 2}^{T} \\
\vdots & \vdots & \ddots & \vdots & \vdots & \vdots & \vdots & \ddots & \vdots \\
\underbrace{\mathbf{0}_{1 \times 2}^{T}}_{(m+p-1) \times(2 m+2 p-2)} & \mathbf{0}_{1 \times 2}^{T} & \ldots & \overline{\mathbf{S}}_{1 \times 2}^{T} & \underbrace{\mathbf{0}_{1 \times 3}^{T}}_{(m+p-1) \times 3} & \underbrace{\mathbf{0}_{1 \times 2}^{T}}_{(m+p-1) \times(2 m+2 p-2)} & \mathbf{0}_{1 \times 2}^{T} & \ldots & \overline{\mathbf{S}}_{1 \times 2}^{T}
\end{array}\right]
$$

the nodal force vector $\{\overline{\mathbf{P}}\}$ is given by

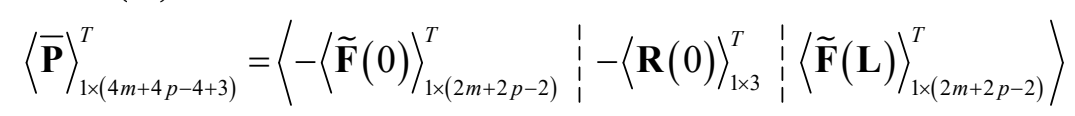

and

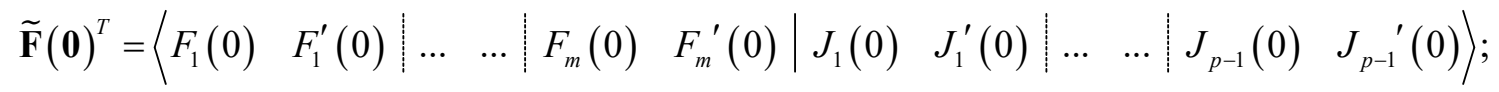



It is possible to express vector $\mathbf{R}(0)_{3 \times 1}$ in term of the nodal force vector $\{\overline{\mathbf{P}}\}$ as

$$
\mathbf{R}(0)_{3 \times 1}=\Lambda_{\mathbf{R} 3 \times(4 m+4 p-4+3)}\{\overline{\mathbf{P}}\}_{(4 m+4 p-4+3) \times 1}
$$


where $\Lambda_{\mathbf{R} 3 \times(4 m+4 p-4+3)}=\left[\begin{array}{lll}\mathbf{0}_{3 \times(2 m+2 p-2)} & -\mathbf{I}_{3 \times 3} & \mathbf{0}_{3 \times(2 m+2 p-2)}\end{array}\right]$ and $\mathbf{I}$ is the identity matrix. From Eqs. (15) and (19), by substituting into Eqs. (6), one obtains the following expressions for the stress fields

$$
\begin{aligned}
\sigma_{z}(y, z) & =\overline{\mathbf{P}}^{T} \mathbf{X}_{\sigma z}+\sigma_{z}^{*}(y, z) ; \\
\tau(y, z) & =\overline{\mathbf{P}}^{T} \mathbf{X}_{\tau}+\tau^{*}(y, z) ; \\
\sigma_{y}(y, z) & =\overline{\mathbf{P}}^{T} \mathbf{X}_{\sigma y}+\sigma_{y}^{*}(y, z)
\end{aligned}
$$

in which the following vectors have been defined:

$$
\begin{aligned}
& \left\{\mathbf{X}_{\sigma z}\right\}=\boldsymbol{\Lambda}_{\mathbf{F}}(z)^{T} \mathbf{a}_{\mathbf{1}}(y)+\boldsymbol{\Lambda}_{\mathbf{F}}(0)^{T} \mathbf{a}_{2}(y)+\boldsymbol{\Lambda}_{\mathbf{F}}{ }^{\prime}(0)^{T} \mathbf{a}_{\mathbf{3}}(y, z)+\Lambda_{\mathbf{R}} \mathbf{b}_{\mathbf{1}}(y, z) \\
& \left\{\mathbf{X}_{\sigma y}\right\}=\boldsymbol{\Lambda}_{\mathbf{F}}{ }^{\prime \prime}(z)^{T} \mathbf{a}_{\mathbf{6}}(y) ; \quad\left\{\mathbf{X}_{\tau}\right\}=\boldsymbol{\Lambda}_{\mathbf{F}}{ }^{\prime}(z)^{T} \mathbf{a}_{\mathbf{4}}(y)+\boldsymbol{\Lambda}_{\mathbf{F}}{ }^{\prime}(0)^{T} \mathbf{a}_{\mathbf{5}}(y)+\Lambda_{\mathbf{R}} \mathbf{b}_{\mathbf{2}}(y)^{T}
\end{aligned}
$$

\subsection{Variational Principle}

The total complementary energy $\pi^{*}$ is the sum of the total complementary strain energy $U^{*}$ and the load potential $V^{*}$ gained by the external forces and tractions, i.e.,

\subsubsection{Complementary strain energy}

$$
\pi^{*}=U^{*}+V^{*}
$$

The total complementary strain energy $U^{*}\left(\sigma_{z}, \sigma_{y}, \tau\right)$ is contributed by longitudinal normal stresses, transversal normal stresses, and transverse shear stresses which can be expressed as

$$
U^{*}=\sum_{j=1}^{p} \frac{1}{2} \int_{L} \int_{A_{j}}\left\langle\varepsilon_{z} \quad \varepsilon_{y} \quad \gamma\right\rangle_{j}\left\{\begin{array}{c}
\sigma_{z} \\
\sigma_{y} \\
\tau
\end{array}\right\} d A_{j} d z
$$

where $j=1,2, \ldots, p$ denotes the $j^{\text {th }}$ layer of the material. For each material, the strains are related to stresses via the generalized 2D Hooke's law, i.e.,

$$
\left\{\begin{array}{l}
\varepsilon_{z} \\
\varepsilon_{y} \\
\gamma
\end{array}\right\}_{j}=\left[\begin{array}{ccc}
1 / E_{z} & -\mu_{y} / E_{y} & 0 \\
-\mu_{z} / E_{z} & 1 / E_{y} & 0 \\
0 & 0 & 1 / G
\end{array}\right]_{j}\left\{\begin{array}{c}
\sigma_{z} \\
\sigma_{y} \\
\tau
\end{array}\right\}
$$

It is noted that for orthotropic materials, the condition $\mu_{z y} / E_{z}=\mu_{y z} / E_{y}$ must be satisfied, i.e., $\mu_{z y}$ and $\mu_{y z}$ are dependent parameters (e.g., Wu and Jensen [16]). From Eqs. (24), by substituting into Eq.(23), one obtains

$$
U^{*}=\sum_{j=1}^{p} \frac{1}{2} \int_{L} \int_{A_{j}}\left\langle\sigma_{z} \quad \sigma_{y} \quad \tau\right\rangle[\mathbf{D}]_{j}\left\{\begin{array}{c}
\sigma_{z} \\
\sigma_{y} \\
\tau
\end{array}\right\} d A_{j} d z ; \quad[\mathbf{D}]_{j}=\left[\begin{array}{ccc}
1 / E_{z} & -\mu_{z y} / E_{y} & 0 \\
-\mu_{y z} / E_{z} & 1 / E_{y} & 0 \\
0 & 0 & 1 / G
\end{array}\right]_{j}
$$


From Eqs. (20), by substituting into Eq. (25), the total complementary strain energy is expressed as

$$
\begin{aligned}
& U^{*}=\sum_{j=1}^{p} \frac{1}{2} \int_{L} \int_{A_{j}} \overline{\mathbf{P}}^{T}\left\langle\mathbf{X}_{\sigma z} \quad \mathbf{X}_{\sigma y} \quad \mathbf{X}_{\tau}\right\rangle[\mathbf{D}]_{j}\left\{\begin{array}{c}
\mathbf{X}_{\sigma z}^{T} \\
\mathbf{X}_{\sigma y}^{T} \\
\mathbf{X}_{\tau}^{T}
\end{array}\right\} \overline{\mathbf{P}} d A_{j} d z+\sum_{j=1}^{p} \int_{L} \int_{A_{j}} \overline{\mathbf{P}}^{T}\left\langle\mathbf{X}_{\sigma z} \quad \mathbf{X}_{\sigma y} \quad \mathbf{X}_{\tau}\right\rangle \frac{1}{2}\left[\mathbf{D}+\mathbf{D}^{T}\right]_{j}\left\{\begin{array}{c}
\sigma_{z}^{*}(y, z) \\
\sigma_{y}^{*}(y, z) \\
\tau^{*}(y, z)
\end{array}\right\} d A_{j} d z+ \\
& +\sum_{j=1}^{p} \frac{1}{2} \int_{L} \int_{A_{j}}\left\langle\sigma_{z}^{*}(y, z) \quad \sigma_{y}^{*}(y, z) \quad \tau^{*}(y, z)\right\rangle[\mathbf{D}]_{j}\left\{\begin{array}{c}
\sigma_{z}^{*}(y, z) \\
\sigma_{y}^{*}(y, z) \\
\tau^{*}(y, z)
\end{array}\right\} d A_{j} d z
\end{aligned}
$$

\subsubsection{Load potential energy}

The load potential gain is the sum of products of stress resultants with corresponding governing displacements, i.e.

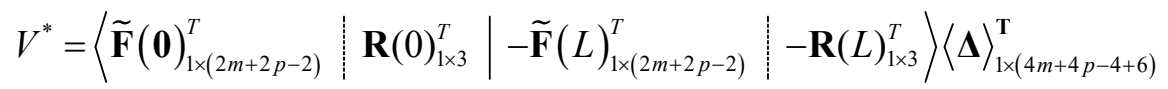

in which

$$
\langle\boldsymbol{\Delta}\rangle^{\mathbf{T}}=\left\langle\langle\tilde{\mathbf{d}}(0)\rangle_{1 \times(2 m+2 p-2)}^{\mathbf{T}}: W(0)|V(0): \theta(0)|\langle\tilde{\mathbf{d}}(L)\rangle_{1 \times(2 m+2 p-2)}^{\mathbf{T}}: W(L) \mid V(L): \theta(L)\right\rangle
$$

with

$$
\begin{aligned}
& \tilde{\mathbf{d}}(0)^{\mathbf{T}}=\left\langle\begin{array}{lll|ll|lll|l|l|l|l}
d_{1}(0) & d_{1}{ }^{*}(0) & \ldots & \ldots & d_{m}(0) & d_{m}{ }^{*}(0) & w_{1}(0) & w_{1}^{*}(0) & \ldots & \ldots & w_{p-1}(0) & w_{p-1}^{*}(0)
\end{array} ; ;\right.
\end{aligned}
$$

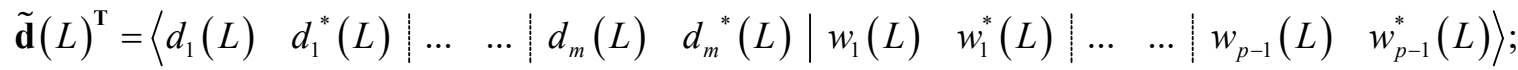

where $d_{i}\left(z_{0}\right)\left(z_{0}=0\right.$ or $L$ and $\left.i=1,2, \ldots, m\right)$ is the displacement conjugate to $F_{i}\left(z_{0}\right)$ while $d_{i}^{*}\left(z_{0}\right)$ is the displacement conjugate to $F_{i}^{\prime}\left(z_{0}\right)$. In Eq. (27), the vector of internal forces at end $z=L$ is defined as $\mathbf{R}(L)^{T}=\langle N(L) \quad Q(L) \quad M(L)\rangle$ (Figure 5). By adopting the internal force definitions $\quad \mathbf{R}(L)^{T}=\langle N(L) \quad Q(L) \quad M(L)\rangle=\int_{A}\left\langle\sigma_{z}(y, L) \quad \tau(y, L) \quad y \sigma_{z}(y, L)\right\rangle d A \quad$ and substituting from the statically admissible stress fields in Eq. (6) one obtains

$$
\begin{aligned}
& \mathbf{R}(L)^{\mathbf{T}}=\int_{A}\left\langle\mathbf{a}_{2}(y)|0| y \mathbf{a}_{2}(y)\right\rangle d A \mathbf{F}(0)+\int_{A}\left\langle\mathbf{a}_{3}(y, L)\left|\mathbf{a}_{\mathbf{5}}(y)\right| y \mathbf{a}_{3}(y, L)\right\rangle d A \mathbf{F}^{\prime}(0)
\end{aligned}
$$

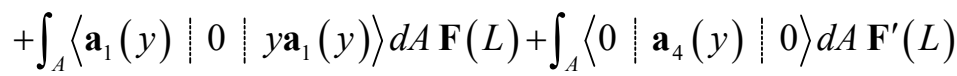

$$
\begin{aligned}
& +\int_{A}\left\langle\mathbf{b}_{1}(y, L)\left|\mathbf{b}_{2}(y)\right| y \mathbf{b}_{1}(y, L)\right\rangle d A \mathbf{R}(0)+\int_{A}\left\langle\sigma_{z}^{*}(y, L)\left|\tau^{*}(y, L)\right| y \sigma_{z}^{*}(y, L)\right\rangle d A
\end{aligned}
$$

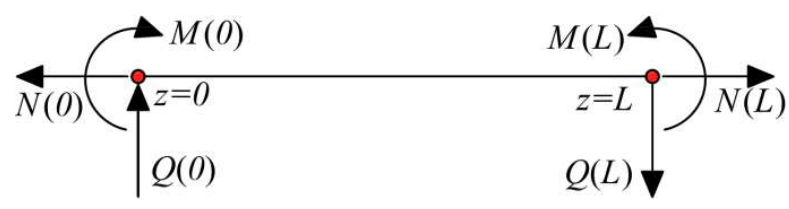

(a)

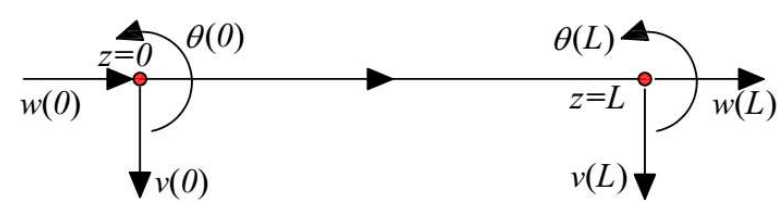

(b) 
Figure 5. Beam sign convention (a) Applied loads and end stress resultants forces, and (b) Energy conjugate end displacements

\subsubsection{Variation of total complementary strain energy}

From Eq.(26), the variation of the total complementary strain energy is given by

$$
\delta U^{*}=\delta \overline{\mathbf{P}}^{\mathbf{T}}\left(\mathbf{H} \overline{\mathbf{P}}+\mathbf{d}_{0}\right)
$$

in which $[\mathbf{H}]_{(4 m+4 p-4+3) \times(4 m+4 p-4+3)}$ is the flexibility matrix defined as

$$
[\mathbf{H}]=\sum_{j=1}^{p} \int_{L} \int_{A_{j}}\left\langle\mathbf{X}_{\sigma z} \quad \mathbf{X}_{\sigma y} \quad \mathbf{X}_{\tau}\right\rangle[\mathbf{D}]_{j}\left\{\begin{array}{c}
\mathbf{X}_{\sigma z}^{T} \\
\mathbf{X}_{\sigma y}^{T} \\
\mathbf{X}_{\tau}^{T}
\end{array}\right\} d A_{j} d z
$$

and $\mathbf{d}_{0}$ the energy conjugate generalized displacement vector given by

$$
\mathbf{d}_{0}=\sum_{j=1}^{p} \int_{L} \int_{A_{j}}\left\langle\mathbf{X}_{\sigma z} \quad \mathbf{X}_{\sigma y} \quad \mathbf{X}_{\tau}\right\rangle \frac{1}{2}\left[\mathbf{D}+\mathbf{D}^{T}\right]_{j}\left\{\begin{array}{l}
\sigma_{z}^{*}(y, z) \\
\sigma_{y}^{*}(y, z) \\
\tau^{*}(y, z)
\end{array}\right\} d A_{j} d z
$$

\subsubsection{Variation of load potential energy}

From Eq. (15), by substituting into Eq. (30), one obtains

$$
\mathbf{R}(L)=\left\{\begin{array}{c}
N(L) \\
Q(L) \\
M(L)
\end{array}\right\}=\left\{\begin{array}{l}
\overline{\mathbf{P}}^{T} \boldsymbol{\Gamma}_{N}(L) \\
\overline{\mathbf{P}}^{T} \boldsymbol{\Gamma}_{Q}(L) \\
\overline{\mathbf{P}}^{T} \boldsymbol{\Gamma}_{M}(L)
\end{array}\right\}+\int_{A}\left\{\begin{array}{c}
\sigma_{z}^{*}(y, L) \\
\tau^{*}(y, L) \\
y \sigma_{z}^{*}(y, L)
\end{array}\right\} d A
$$

in which the following vectors are defined

$$
\begin{aligned}
& \boldsymbol{\Gamma}_{N}(L)_{(4 m+4 p-4+3) \times 1}=\int_{A}\left[\boldsymbol{\Lambda}_{\mathbf{F}}(L)^{T} \mathbf{a}_{\mathbf{1}}(y)+\boldsymbol{\Lambda}_{\mathbf{F}}(0)^{T} \mathbf{a}_{\mathbf{2}}(y)+\boldsymbol{\Lambda}_{\mathbf{F}}{ }^{\prime}(0)^{T} \mathbf{a}_{\mathbf{3}}(y, L)+\Lambda_{\mathbf{R}}(0)^{T} \mathbf{b}_{\mathbf{1}}(y, L)\right] d A \\
& \boldsymbol{\Gamma}_{Q}(L)_{(4 m+4 p-4+3) \times 1}=\int_{A}\left[\boldsymbol{\Lambda}_{\mathbf{F}}{ }^{\prime}(L)^{T} \mathbf{a}_{\mathbf{4}}(y)+\boldsymbol{\Lambda}_{\mathbf{F}}{ }^{\prime}(0)^{T} \mathbf{a}_{\mathbf{5}}(y)+\Lambda_{\mathbf{R}}(0)^{T} \mathbf{b}_{\mathbf{2}}(y)\right] d A \\
& \boldsymbol{\Gamma}_{M}(L)_{(4 m+4 p-4+3) \times 1}=\int_{A} y\left[\boldsymbol{\Lambda}_{\mathbf{F}}(L)^{T} \mathbf{a}_{\mathbf{1}}(y)+\boldsymbol{\Lambda}_{\mathbf{F}}(0)^{T} \mathbf{a}_{\mathbf{2}}(y)+\boldsymbol{\Lambda}_{\mathbf{F}}{ }^{\prime}(0)^{T} \mathbf{a}_{\mathbf{3}}(y, L)+\Lambda_{\mathbf{R}}(0)^{T} \mathbf{b}_{\mathbf{1}}(y, L)\right] d A
\end{aligned}
$$

Equation (34) can be used to eliminate $\mathbf{R}(L)$ from Eq.(27) yielding

$$
\begin{aligned}
V^{*}= & -\overline{\mathbf{P}}_{1 \times(4 m+4 p-4+3)}^{\mathbf{T}}[\boldsymbol{\Gamma}(L)]_{(4 m+4 p-4+3) \times(4 m+4 p-4+6)}\{\boldsymbol{\Delta}\}_{(4 m+4 p-4+6) \times 1} \\
& -\left\langle\int_{A} \sigma_{z}^{*}(y, L) d A: \int_{A} \tau^{*}(y, L) d A: \int_{A} y \sigma_{z}^{*}(y, L) d A\right\rangle\left\{\begin{array}{c}
W(L) \\
V(L) \\
\theta(L)
\end{array}\right\}
\end{aligned}
$$

in which

$$
\boldsymbol{\Gamma}(L)=\left[\begin{array}{llll}
\mathbf{I}_{(4 m+4 p-4+3) \times(4 m+4 p-4+3)} & \boldsymbol{\Gamma}_{N}(L)_{(4 m+4 p-4+3) \times 1} & \boldsymbol{\Gamma}_{Q}(L)_{(4 m+4 p-4+3) \times 1} & \boldsymbol{\Gamma}_{M}(L)_{(4 m+4 p-4+3) \times 1}
\end{array}\right]
$$

From Eq. (35), by taking variations with respect to the nodal force vector $\overline{\mathbf{P}}^{\mathrm{T}}$, one obtains 


$$
\delta V^{*}=-\delta \overline{\mathbf{P}}_{1 \times(4 m+4 p-4+3)}^{T}[\boldsymbol{\Gamma}(L)]_{(4 m+4 p-4+3) \times(4 m+4 p-4+6)}\{\boldsymbol{\Delta}\}_{(4 m+4 p-4+6) \times 1}
$$

In summary, from Eqs. (31) and (37), the stationarity condition is expressed by setting the variation of total complementary potential energy to zero, yielding

$$
\delta \pi^{*}=\delta U^{*}+\delta V^{*}=\delta \overline{\mathbf{P}}^{\mathrm{T}}\left[\left(\mathbf{H} \overline{\mathbf{P}}+\mathbf{d}_{0}\right)-\boldsymbol{\Gamma}(L) \boldsymbol{\Delta}\right]=0
$$

\subsubsection{Augmented form of the finite element formulation}

Equation (38) is used to solve for the nodal force vector yielding

$$
\overline{\mathbf{P}}=\mathbf{H}^{-1}[\boldsymbol{\Gamma}(L)] \Delta-\mathbf{H}^{-1} \mathbf{d}_{0}
$$

It is noted that the size of nodal force vector $\overline{\mathbf{P}}_{(4 m+4 p-4+3) \times 1}$ is smaller than that of the nodal displacement vector $\boldsymbol{\Delta}_{(4 m+4 p-4+6) \times 1}$. It is thus required to augment nodal force vector $\overline{\mathbf{P}}$ by combining it with the vector of internal forces $\mathbf{R}(L)$. From Eq. (39), by substituting into Eqs. (34), one obtains

$$
\mathbf{R}(L)=\left\{\begin{array}{l}
\left\langle\boldsymbol{\Gamma}_{N}^{T}(L)\right\rangle_{1 \times(4 m+4 p-4+3)} \\
\left\langle\boldsymbol{\Gamma}_{Q}^{T}(L)\right\rangle_{1 \times(4 m+4 p-4+3)} \\
\left\langle\boldsymbol{\Gamma}_{M}^{T}(L)\right\rangle_{1 \times(4 m+4 p-4+3)}
\end{array}\right\}\left[\mathbf{H}^{-1}\langle\boldsymbol{\Gamma}(L)\rangle \boldsymbol{\Delta}-\mathbf{H}^{-1} \mathbf{d}_{0}\right]+\int_{A}\left\{\begin{array}{c}
\sigma_{z}^{*}(y, L) \\
\tau^{*}(y, L) \\
y \sigma_{z}^{*}(y, L)
\end{array}\right\} d A
$$

From Eq. (40), by combining with Eq. (39) into a single matrix, the augmented nodal force vector is given as

$$
\{\mathbf{P}\}_{(4 m+4 p-4+6) \times 1}=[\mathbf{K}]_{(4 m+4 p-4+6) \times(4 m+4 p-4+6)}\{\boldsymbol{\Delta}\}_{(4 m+4 p-4+6) \times 1}-\left\{\mathbf{P}_{0}\right\}_{(4 m+4 p-4+6) \times 1}
$$

in which

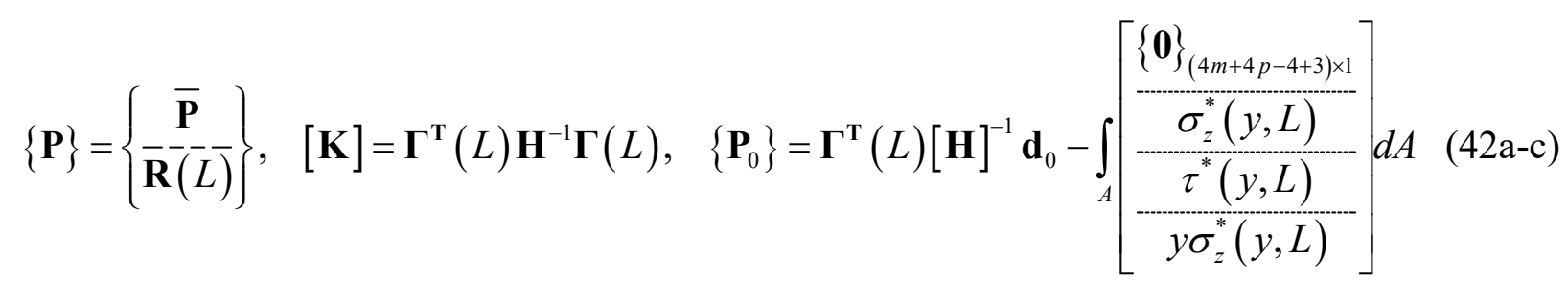

where $\{\mathbf{P}\}$ is the augmented nodal force vector, $[\mathbf{K}]$ is the stiffness matrix, and $\left\{\mathbf{P}_{0}\right\}$ is the energy equivalent load vector. The steps for forming $[\mathbf{K}]$ and $\left\{\mathbf{P}_{0}\right\}$ are provided in Appendix B. In general, the size of matrix $[\mathbf{K}]$ and vector $\left\{\mathbf{P}_{0}\right\}$ will depend on the reduced number of stress terms $m=n-2$ and the number of layers $p$ (as defined in Eq. 1). The entries of the matrix depend on the constitutive constants $E_{z}, E_{y}, G, \mu_{z y}, \mu_{y z}$ for each layer. Thus, explicit expressions for the entries of $[\mathbf{K}]$ and $\left\{\mathbf{P}_{0}\right\}$ are too lengthy to provide. Instead, Appendix C provides, as an illustrative 
example, the expressions for $[\mathbf{H}],[\mathbf{K}]$ and $\left\{\mathbf{P}_{0}\right\}$ for the special case of a homogeneous beam element with a rectangular cross-section made of an orthotropic material when the number of terms is taken as $n=3$.

\section{Verification and Applications}

The present section aims at assessing the validity of the present finite element formulation in predicting deflections and stresses for both homogeneous and multilayer beams and showcasing its applicability to a variety of practical problem. While the formulation is primarily intended for multilayer beams, it can also be applied for homogeneous beams by eliminating all Heaviside Step Functions terms in the second summation of Eq. (1) and subsequent equations, by setting $p=1$. The first two examples provide an assessment of the validity of the present theory for the case of homogeneous beams. Examples 3-6 then illustrate the applicability of the theory to multilayer beams including wood beams and steel beams, both strengthened with GFRP plates, and sandwich beams. In all cases, three-dimensional finite element analyses under ABAQUS are provided as benchmark solutions to assess the validity of the results.

\section{Verification Example 1. Simply supported homogeneous beam under uniform traction}

The example in Carrera and Giunta [17] is revisited in the present study. A simply supported beam with a slender rectangular cross-section is considered. The beam is subjected to a transversely uniform traction $\sigma_{y}(-h / 2, z)=\sigma=1.0 \mathrm{MPa}$ acting at the top surface (Figure 6) while other applied tractions $\tau(-h / 2, z), \tau(h / 2, z), \sigma_{y}(h / 2, z)$ vanish. Material is steel with a modulus of elasticity $E=200,000 \mathrm{MPa}$ and a Poison's ratio $\mu=0.3$. Beam depth is $1.0 \mathrm{~m}$. The depth to width ratio $h / b$ of the cross-section is 100 while span to depth $L / h$ is varied from 2 to 50. It is assumed that the beam is laterally restrained to avoid lateral buckling.

Convergence study: A convergence study is conducted for the case $L / h=5$ (Figure 7 ). For a homogeneous beam, the number of layers is set to $p=1$ and the second summation in Eq. (1) vanishes. For $n=3$, Eq. (1) takes the form $\sigma_{z}(y, z)=F_{1}(z)+y F_{2}(z)+y^{2} F_{3}(z)$ while for $n=4$, Eq. (1) becomes $\sigma_{z}(y, z)=F_{1}(z)+y F_{2}(z)+y^{2} F_{3}(z)+y^{3} F_{4}(z)$. Figure $7 \mathrm{a}$ and $7 \mathrm{~b}$ presents the deflection predicted by the present theory when the number of stress terms is taken as $n=3$ and $n=4$, respectively. The number of elements based on the present finite element formulation are varied from 2 to 10 . The predicted peak deflection is observed to be independent of the number 
of elements taken. This is due to the fact that the cubic shape functions assumed to interpolate functions $F_{i}(z)$ in Eqs. (13) are enough to capture the closed form solution of the compatibility equations. Similar observations are made for any number of stress terms $n$. The peak deflection for $n=3$ is $0.5331 \mathrm{~mm}$ while that based on $n=4$ is $0.5328 \mathrm{~mm}$, a negligible $0.1 \%$ difference. Further increase in $n$ was found not to change the predicted peak deflection. Of particular interest is to note that the predicted deflection decreases as $n$ increases. Table 1 provides the normal stresses $\left(\sigma_{z, \max }\right)$ at the mid-span extreme fiber and the maximum shear stresses $\left(\tau_{\max }\right)$ at section mid-height of section at the support. For $n=3$, both stresses are observed to be independent of the number of elements. In contrast, for $n=4$, six elements are needed for the normal stresses to attain convergence, albeit only two elements were needed for the shear stresses to converge. In summary, convergence of both stresses and displacements is attained when six elements are taken.

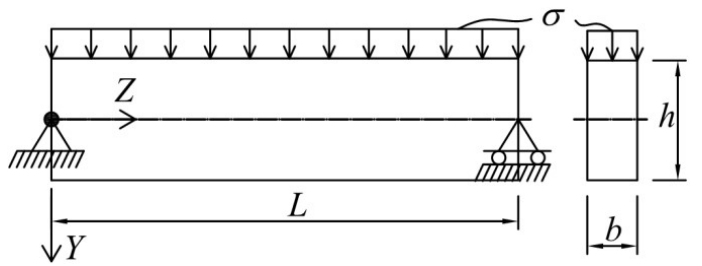

Figure 6. Simply supported beam under a uniform traction

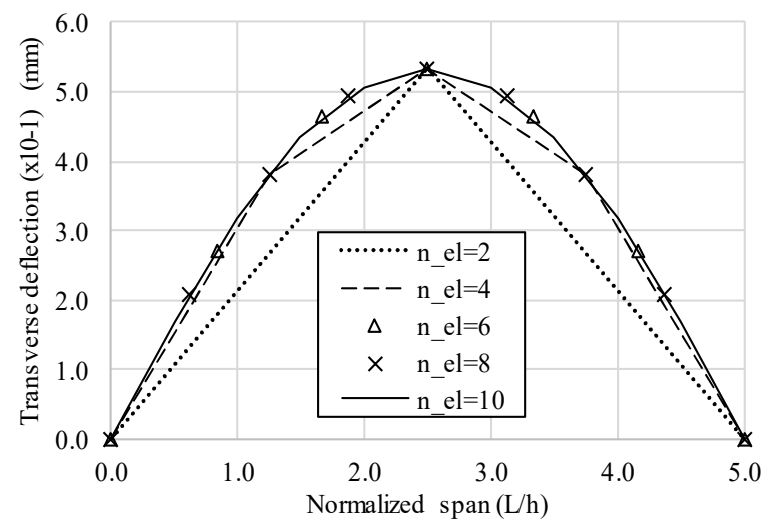

(a) $n=3$

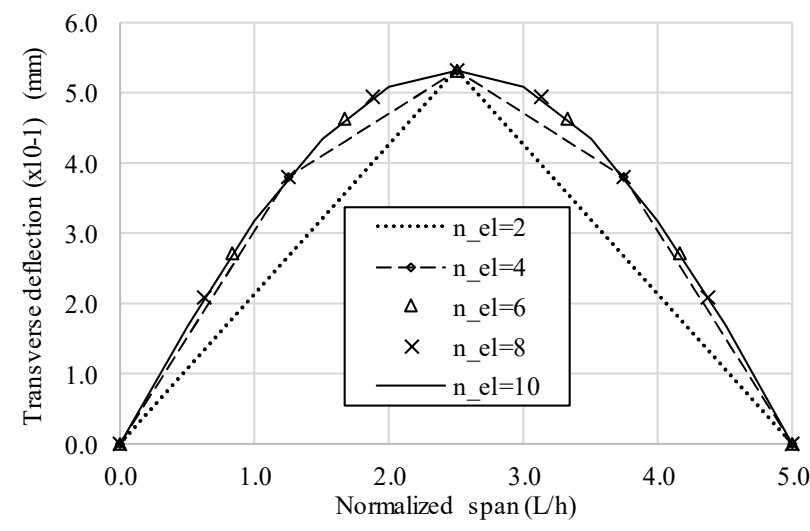

(b) $n=4$

Figure 7. Mesh sensitivities for deflection obtained from present finite element formulation

Table 1. Mesh sensitivity study for peak stresses (MPa)

\begin{tabular}{cccccc} 
Number of & \multicolumn{2}{c}{$\mathrm{n}=3$} & & \multicolumn{2}{c}{$\mathrm{n}=4$} \\
\cline { 2 - 3 } \cline { 5 - 6 } elements & $\sigma_{z, \max }$ & $\tau_{\max }$ & & $\sigma_{z, \max }$ & $\tau_{\max }$ \\
\cline { 2 - 3 } 2 & 18.75 & 3.75 & & 16.99 & 3.71 \\
4 & 18.75 & 3.75 & & 18.79 & 3.71 \\
6 & 18.75 & 3.75 & & 18.95 & 3.71 \\
8 & 18.75 & 3.75 & & 18.96 & 3.71
\end{tabular}




\begin{tabular}{lllll}
10 & 18.75 & 3.75 & 18.96 & 3.71 \\
\hline
\end{tabular}

Comparative study: The peak deflection and stresses based on the present solution (Table 2) are compared to the those based on the Euler Bernouilli beam (EB), the Timoshenko beam (TB) solution based on a shear coefficient $\kappa=0.85$ [2], the high-order solution by Carrera and Giunta [17], and the elasticity solution in Timoshenko and Goodier [26], referred to as TG1970. The relevant equations in TG1970 are $v=\left[1+12 c^{2}(4 / 5+\mu / 2) / 5 L_{t}^{2}\right] 5 q L_{t}^{4} / 24 E I_{t}$, $\sigma_{z}=q\left(L_{t}^{2}-z_{t}^{3}\right) y_{t} / 2 I_{t}+q\left(5 y_{t}^{3}-3 c^{2} y_{t}\right) / 15 I_{t} ; \sigma_{y}=q\left(c^{2} y_{t}-y_{t}^{3} / 3-2 c^{3} / 3\right) / 2 I_{t} ; \tau_{z y}=q\left(y_{t}^{2}-c^{2}\right) z_{t} / 2 I_{t} ;$ in which $L_{t}=L / 2 ; c=h / 2 ; q=\sigma b$; and $I_{t}=b h^{3} / 12$. The TG1970 solution is taken as a reference solution against which all solutions are compared. For the case $L / h=2$, the solution based on EB grossly underestimates the deflection by $36.2 \%$. The deflection based on TB with $\kappa=0.85$ is $2.5 \%$ higher than that based on the reference solution. The peak deflection predicted based on the present solution with $n=3$ is only $1.0 \%$ larger than that predicted by the reference solution. The present solution with $n=4$ or 5 is in exact agreement with that based on TG1970 solution for all spans. The solution of Carrera and Giunta [17] also provides identical predictions to the TG1970 solution for short beams when the number of terms taken is $n=6$. In this respect, the present solution attains the solution of TG1970 with fewer terms than that in Carrera and Giunta [17]. As expected, for higher $L / h$ ratios, the effect of transverse shear stresses on the deflection becomes less significant and the difference between all solutions become smaller (Table 2).

Table 2. Mid-span deflections of the simply supported rectangle beam under the uniform traction

\begin{tabular}{ccclclcl}
\hline Solution & Order & $\begin{array}{c}\text { Deflection } \\
(\mathrm{mm})\end{array}$ & $\begin{array}{l}\text { Difference } \\
(\%)\end{array}$ & $\begin{array}{c}\text { Deflection } \\
(\mathrm{mm})\end{array}$ & $\begin{array}{l}\text { Difference } \\
(\%)\end{array}$ & $\begin{array}{c}\text { Deflection } \\
(\mathrm{m})\end{array}$ & $\begin{array}{l}\text { Difference } \\
(\%)\end{array}$ \\
\hline EB & $\mathrm{NA}$ & 0.0125 & 36.2 & 0.4883 & 8.1 & 4.88 & 0.2 \\
$\mathrm{~TB}$ & $\mathrm{NA}$ & 0.0201 & 2.5 & 0.5360 & 0.6 & 4.89 & 0.0 \\
$\mathrm{TG} 1970$ & $\mathrm{NA}$ & 0.0196 & 0.0 & 0.5328 & 0.0 & 4.89 & 0.0 \\
\multirow{2}{*}{ Present } & $\mathrm{n}=3$ & 0.0198 & 1.0 & 0.5331 & 0.1 & 4.89 & 0.0 \\
\multirow{2}{*}{ solution } & $\mathrm{n}=4$ & 0.0196 & 0.0 & 0.5328 & 0.0 & 4.89 & 0.0 \\
& $\mathrm{n}=5$ & 0.0196 & 0.0 & 0.5328 & 0.0 & 4.89 & 0.0 \\
\hline
\end{tabular}

$\%$ difference of row $\mathrm{i}=$ (deflection at row i-deflection of TG1970 solution)*100/( deflection of TG1970 solution).

Figure $8 \mathrm{a}$ and $8 \mathrm{~b}$ present the longitudinal normal stress distributions along the section normalized height for span-to-height ratios of $L / h=2$ and $L / h=5$ while Figure $8 \mathrm{c}, \mathrm{d}$ present the transverse normal and shear stresses for $L / h=5$. For $L / h=2$ (Figure 8a), the present solution with $n=4$ 
depicts a slightly nonlinear distribution of the longitudinal normal stresses with depth, in a manner consistent with the TG1970 solution. In contrast, both the EB and the present solutions with $n=3$ predict a linear distribution for the stresses. For the case $L / h=5$ (Figure $8 \mathrm{~b}-\mathrm{d}$ ), the longitudinal normal and transverse shear stresses are observed to be identical for the solutions based on EB, TG1970 and present solution (with $n=3,4$ ). Contrary to the EB solution, which does not capture the transverse normal stresses, the TG1970 and present solutions depict non-zero transverse normal stresses (Figure 8c). Unlike the case $L / h=2$, excellent stress predictions can be obtained for $L / h=5$ when the number of terms taken is $n=3$.

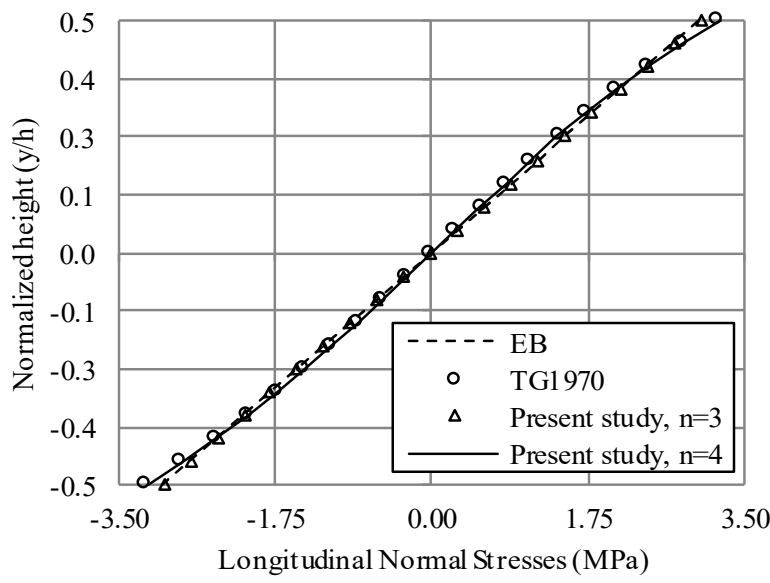

(a) $L / h=2$

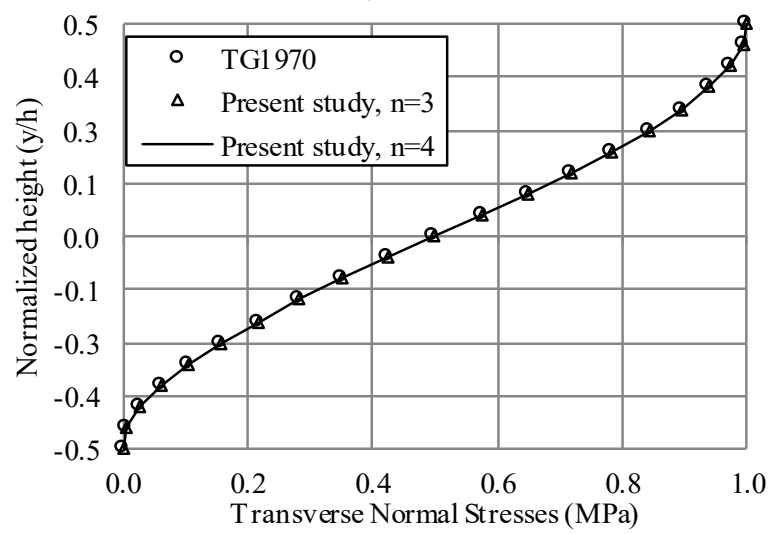

(c) $L / h=5$

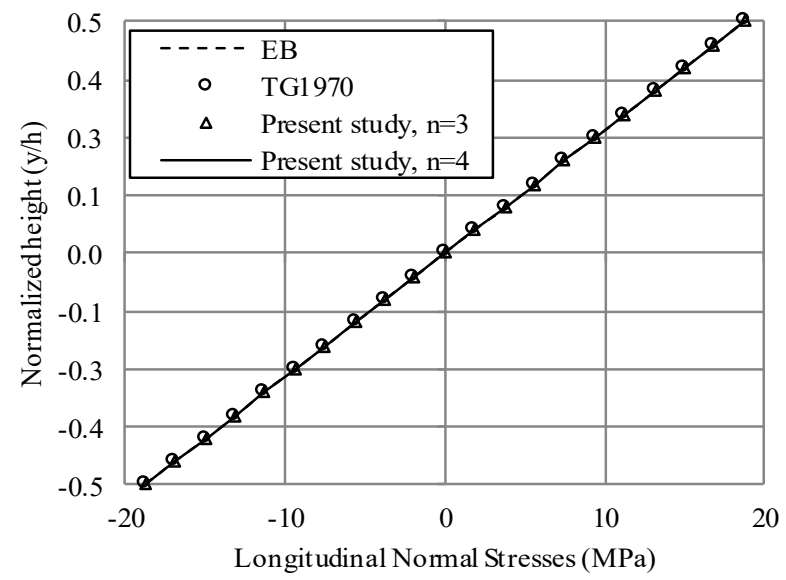

(b) $L / h=5$

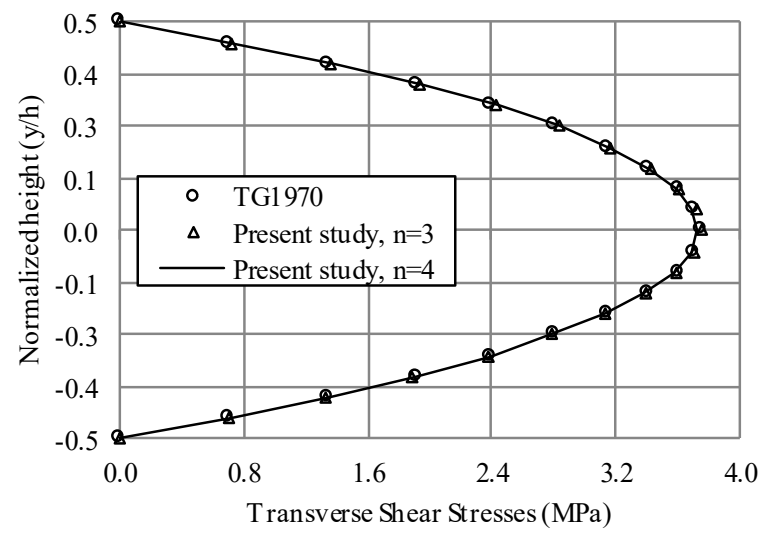

(d) $L / h=5$ 
Figure 8. Distribution across the normalized cross-section height ( $y / h$ ) of: (a) Longitudinal normal stresses at $z=L / 2$ in case of $L / h=2$, (b) Longitudinal normal stresses at mid-span $z=L / 2$, (c) Transverse normal stresses at $z=0$ and (d) Transverse shear stresses at $z=0$ in cases of $L / h=5$.

\section{Verification Example 2. Clamped homogeneous beam with Tee cross-section}

While Example 1 focused on a beam with a rectangular cross-section, the present example showcases the ability of the theory to model a mono-symmetrical cross-section. A steel beam is clamped at both ends and subjected to a vertical uniform traction $\sigma=1.0 \mathrm{MPa}$ acting on the top surface

Figure 9a,b). The beam cross-section is a T-shaped with the dimensions shown in Fig. 9b. Three span-to-depth ratios $L / h=2,5,10$ are considered where $h$ denotes the cross-sectional depth. The modulus of elasticity of steel is 200GPa and its Poison's ratio is 0.3 . It is required to compare the mid-span deflection predicted by the present solution, the Euler-Bernoulli (EB) beam, Timoshenko beam (TB) and 3D-FEA under Abaqus.

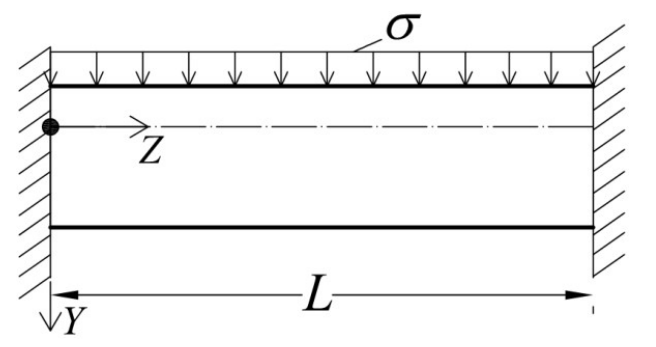

(a)

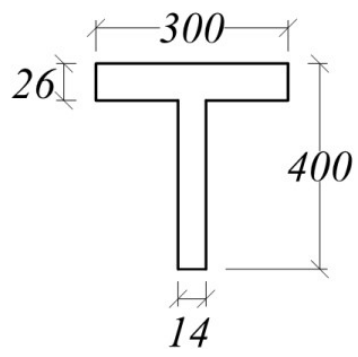

(b)

Figure 9. (a) fixed-fixed beam under uniform traction (b) cross-section for Example 2

In the present solution, all displacements at member ends are set to vanish to model the fixity conditions of the problem. A mesh sensitivity analysis indicated that 6 elements are enough for the deflections to convergence. The Timoshenko beam solution predicts a peak deflection of $v=\sigma b L^{4} / 384 E I_{x x}+\sigma b L^{2} / 8 \kappa G A$ in which shear correction factor $\kappa=0.333$ [2]. The EulerBernoulli solution is obtained from the Timoshenko solution by omitting the shear deformation term. A 3D FEA is conducted under ABAQUS using the eight-nodded continuum element C3D8R with 3 degrees of freedom per node with reduced integration to avoid shear locking. A mesh sensitivity was conducted and the results reported are based on the converged results. Deflection predictions for $n=4$ (Table 3) are observed to match those based on $n=5$. The predictions of the present solution are in better agreement with the 3D FEA than the EB or TM solutions. As discussed in Example 1, the present solution converges to the displacements from 
above and provides a more flexible representation of the problem than the Timoshenko beam, suggesting that: (a) it naturally captures shear deformation effects without needing a shear modification factor, and (b) it incorporates the effect of transverse deformation (captured in the 3D FEA but not in the Timoshenko beam theory).

Table 3. Maximum deflection for clamped homogeneous beams

\begin{tabular}{|c|c|c|c|c|c|c|c|}
\hline \multirow[b]{2}{*}{ Solution } & \multirow[b]{2}{*}{ Order } & \multicolumn{2}{|c|}{$L / h=2$} & \multicolumn{2}{|c|}{$L / h=5$} & \multicolumn{2}{|c|}{$L / h=10$} \\
\hline & & $\begin{array}{l}\text { Deflection } \\
(\mathrm{mm})\end{array}$ & $\begin{array}{l}\text { Difference } \\
(\%)\end{array}$ & $\begin{array}{l}\text { Deflection } \\
\quad(\mathrm{mm})\end{array}$ & $\begin{array}{l}\text { Difference } \\
(\%)\end{array}$ & $\begin{array}{l}\text { Deflection } \\
\quad(\mathrm{mm})\end{array}$ & $\begin{array}{l}\text { Difference } \\
(\%)\end{array}$ \\
\hline EB & $\mathrm{NA}$ & 0.0086 & 87.1 & 0.3346 & 54.8 & 5.354 & 23.2 \\
\hline TB & NA & 0.0804 & 18.6 & 0.7834 & 5.8 & 7.149 & 2.6 \\
\hline 3D FEA & NA & 0.0652 & 2.4 & 0.7327 & 1.0 & 6.961 & 0.1 \\
\hline \multirow{3}{*}{$\begin{array}{c}\text { Present } \\
\text { study }\end{array}$} & $n=3$ & 0.0706 & 5.7 & 0.7569 & 2.2 & 6.971 & 0.0 \\
\hline & $\mathrm{n}=4$ & 0.0668 & 0.0 & 0.7403 & 0.0 & 6.968 & 0.0 \\
\hline & $n=5$ & 0.0668 & 0.0 & 0.7403 & 0.0 & 6.968 & 0.0 \\
\hline
\end{tabular}

\section{Example 3: Application to Wood beam strengthened with single GFRP plate}

A simply supported wood beam with a solid rectangular cross-section $(b \times h=200 \times 200 \mathrm{~mm})$ is considered (Figure 10). The span to depth ratios investigated are $\mathrm{L} / \mathrm{h}$ of 5, 10 and 20. The beam is strengthened with a $9.5 \mathrm{~mm}$-thick GFRP plate through a 1-mm thick adhesive layer (Figure 10). Wood and GFRP are treated as orthotropic materials while the adhesive is considered isotropic (Table 4). Top surface traction $\sigma=0.4 M P a$ is applied to the beam for span $\mathrm{ratios} \mathrm{L} / \mathrm{h}=$ 5 and 10 while the traction value $\sigma=0.05 M P a$ is applied for $\mathrm{L} / \mathrm{h}=20$ (to keep deflections within acceptable limits). It is required to (1) predict the maximum deflection at mid-span, (2) predict the interfacial shear and normal stresses in the adhesive, and (3) obtain the longitudinal normal stress profile at mid-span cross-section based on the present solution and compare results with the 3D FEA solution under ABAQUS.

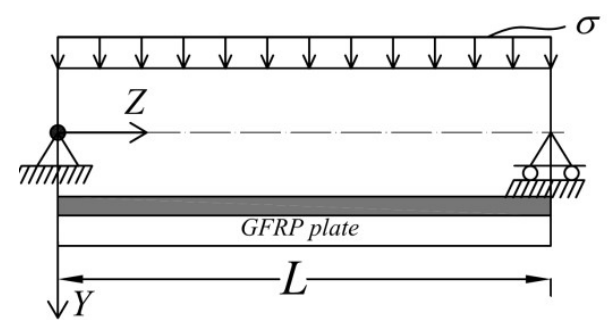

(a)



(b)

Figure 10. Wood beam strengthened with GFRP (a) elevation and (b) cross-section

Table 4. Material properties of a wood beam strengthened with a GFRP plate

Material $E_{z}(\mathrm{GPa}) \quad E_{y}(\mathrm{GPa}) \quad \mu_{z y} \quad G_{y z}(\mathrm{GPa})$




\begin{tabular}{ccccc}
\hline Wood & 11.4 & 1.482 & 0.35 & 1.243 \\
Adhesive & 3.18 & 3.18 & 0.3 & 1.223 \\
GFRP & 19.3 & 8.873 & 0.295 & 2.834 \\
\hline
\end{tabular}

Mesh sensitivity study for the present solution: The case $L / h=10$ is taken to perform a mesh study for the present solution and the 3D FEA solutions. The boundary conditions $W(0)=V(0)=V(L)=0$ are enforced in the present solution. For the present three-layer problem, the number of interfaces is $p-1=2$. The number of stress terms $n$ introduced in Eq. (1) is varied from 3 to 8 and the corresponding longitudinal stresses expression from Eq. (1) take the form $\sigma_{z}(y, z)=\sum_{i=1}^{n} y^{i-1} F_{i}(z)+H(y-h / 2) J_{1}(z)+H\left(y-h / 2-t_{2}\right) J_{2}(z)$ where $n=3,4,5,6,7,8$. In a manner similar to the mesh study conducted for Example 1, the deflections and interfacial stresses convergence is attained with 6 elements for $n=3,8$ elements for $n=4,12$ for $n=5,16$ for $n=6,20$ for $n=7$, and 20 for $n=8$. In order to capture the high gradients for the interfacial stresses near the bond ends, 30 elements per meter long were taken for all runs.

Figure 12a,b depict the interfacial shear and peeling stresses at the adhesive-wood interface based on the present solution. The interfacial stresses based on $n=3$ are found smaller than those based on $\mathrm{n}=5,7$ and 8 . Also, the interfacial stresses are found to converge when $\mathrm{n}=7$.

Mesh sensitivity for 3D FEA solution: A 3D FEA solution based on ABAQUS was conducted for validation. The $3 \mathrm{D}$ mesh adopted is similar to that reported in $[27,28]$. The eight-node brick elements C3D8R is selected from ABAQUS library. The element has eight nodes with three translations per node. To avoid volumetric locking, the element uses reduced integration and thus has a single integration point at the element centroid. The input material properties in the longitudinal and transverse directions are identical to those in Fig. 11b. The elasticity and shear moduli and Poisson's ratio characterizing the material behavior in the lateral direction are taken to conform to those of the transverse direction. Only half of the span is modeled to account for the symmetry of the problem. Three meshes were generated with parameters $n_{1}$ through $n_{5}$ as listed in Figure 11a,b where the number of elements across the cross-sectional width, GFRP plate thickness, adhesive thickness, wood beam depth are $n_{1}$ through $n_{4}$ respectively and the number of elements along the longitudinal direction is $n_{5}$. 


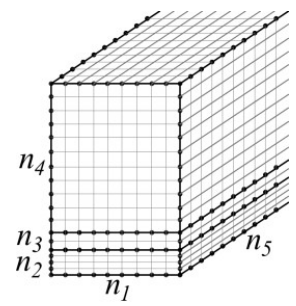

(a)

\begin{tabular}{ccccccc}
\hline Mesh & $n_{1}$ & $n_{2}$ & $n_{3}$ & $n_{4}$ & $n_{5}$ & \#DOF \\
\hline 1 & 10 & 10 & 4 & 10 & 150 & 124,575 \\
2 & 50 & 10 & 4 & 100 & 400 & $2,632,565$ \\
3 & 80 & 10 & 4 & 100 & 500 & $4,807,095$
\end{tabular}

(b)

Figure 11. Section meshing parameters (a) Meshing parameters and (b) Values of parameters

The mesh study for the predicted interfacial shear and peeling stresses is conducted for Point 1 along the longitudinal $z$-direction as shown in Figure 12b,d. The peak interfacial shear stress based on the coarse 3D FEA mesh is $1.48 \mathrm{MPa}$ (Figure 12b) while that based on the medium mesh is $0.98 \mathrm{MPa}$, and that for the fine mesh is $0.97 \mathrm{MPa}$, all occurring at a distance of $120 \mathrm{~mm}$ from the bond end. The interfacial peeling stress distribution based on Mesh 1 exhibits an oscillatory behavior (Figure 12d) while those based on Meshes 2 and 3 practically agree with one another. Within the 3D FEA solution, the mesh size is observed to significantly influence the predicted interfacial shear stresses. Also, the above mesh study suggests that convergence is achieved for Mesh 2.

Comparisons of interfacial shear and peeling stresses: The peak shear and peeling stresses based on the predictions of the present solution with a number of stress terms $n=3$ are $0.80 \mathrm{MPa}$ and 0.09 MPa, respectively, while those based on the 3D FEA solution with Mesh 2 are $0.97 \mathrm{MPa}$ and $0.109 \mathrm{MPa}$, corresponding to $17.5 \%$ and $18.1 \%$ differences, respectively. In contrast, the present solution with $n=7$ predicts a peak shear stress of $0.96 \mathrm{MPa}$ and a peak peeling stress of 0.114 $\mathrm{MPa}$. The converged solutions for $\mathrm{n}=7$ is $1.0 \%$ lower than the shear stresses and $4.4 \%$ higher than the peeling stresses as predicted by the $3 \mathrm{D}$ FEA. 


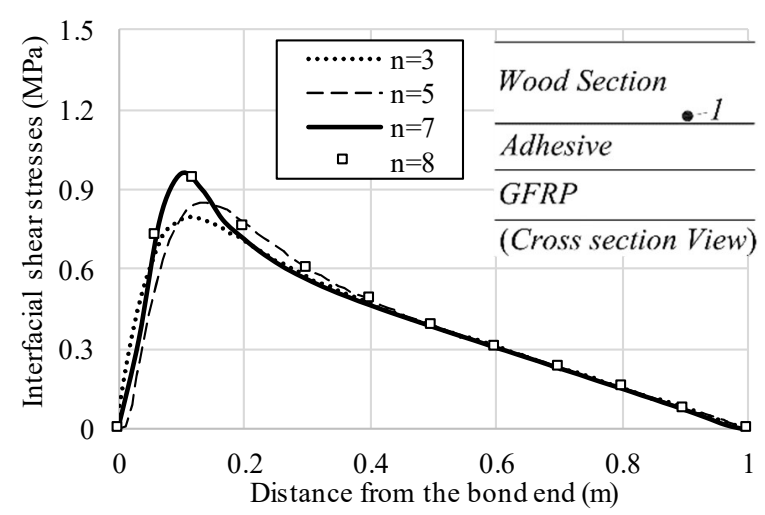

(a) Present solution

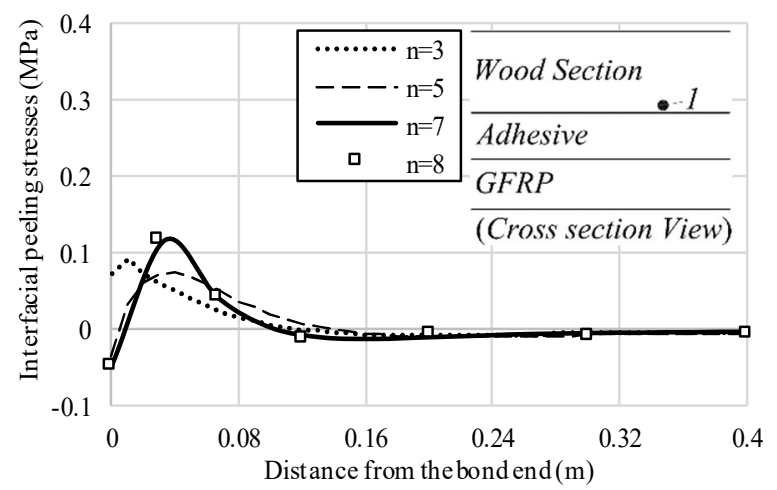

(c) Present solution

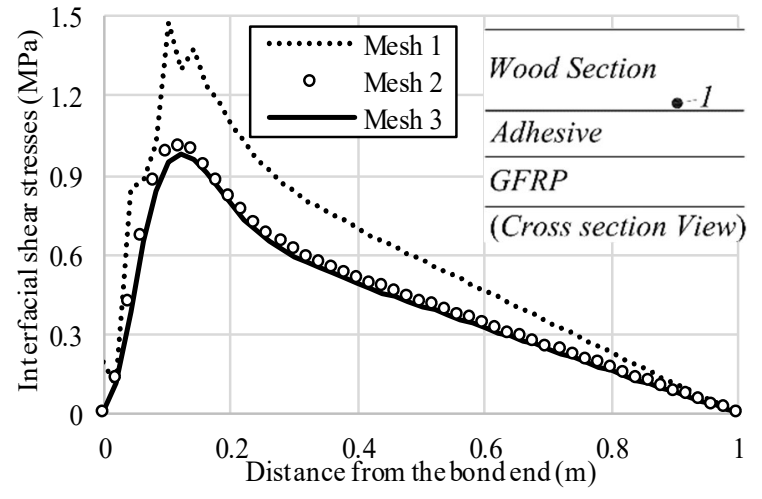

(b) 3D FEA

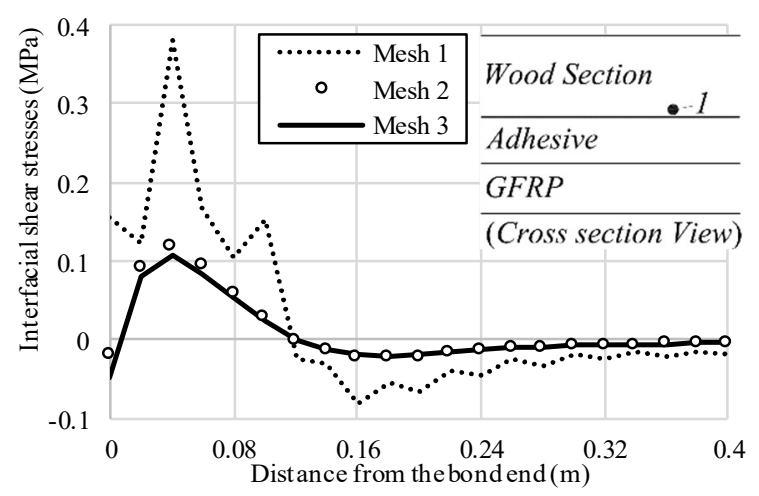

(d) 3D FEA

Figure 12. Mesh studies for the interfacial stresses extracted at Point 1 (a) Interfacial shear stresses - Present solution, (b) Interfacial shear stresses - 3D FEA, (c) Interfacial peeling stresses Present solution, (d) Interfacial peeling stresses- 3D FEA

The previous section focused on the stresses at the upper interface as extracted from the wood section (i.e., point 1 in Figure 12). In principle, satisfying the infinitesimal equilibrium condition at the interfaces necessitates that shear and peeling stresses at points 1 and 2 are equal (Figure 13a-d). A similar argument can be made for points 3 and 4. This indeed is the case under the present solution which is based on satisfying the equilibrium conditions in Eqs. 2(a-b), but is not the case for 3D FEA solution which satisfies equilibrium only in an average integral sense. This is illustrated by considering the peeling and shear stresses based from the present solution with $\mathrm{n}=7$ and the 3D FEA solution based on Mesh 2 (Figure 13a-d). It is observed that (1) the 3D FEA solution predicts an artificial jump in the stresses at the interfaces (i.e., between Points 1 and 2 and between Points 3 and 4) in contrast to the present solution which provides a continuous stress distribution at the interface (e.g., exactly identical stresses at points 1 and 2), (2) among points 14, both solutions predict that the highest stresses take place at Point 1, and (3) the interfacial shear stresses at Points 2 and 3 are found almost identical. The computational time for the 3D FEA solution based on Mesh 2 was 3.75 hours on a computer with two Intel (R) Xeon (R) CPU E5- 
24300 processors at $2.20 \mathrm{GHz}$ speed and $64 \mathrm{~GB}$ of RAM. This compares to 12.2 minutes for the present solution (implemented in a non-compiled MATLAB script file) with $n=7$ and 60elements.



(a) Present solution

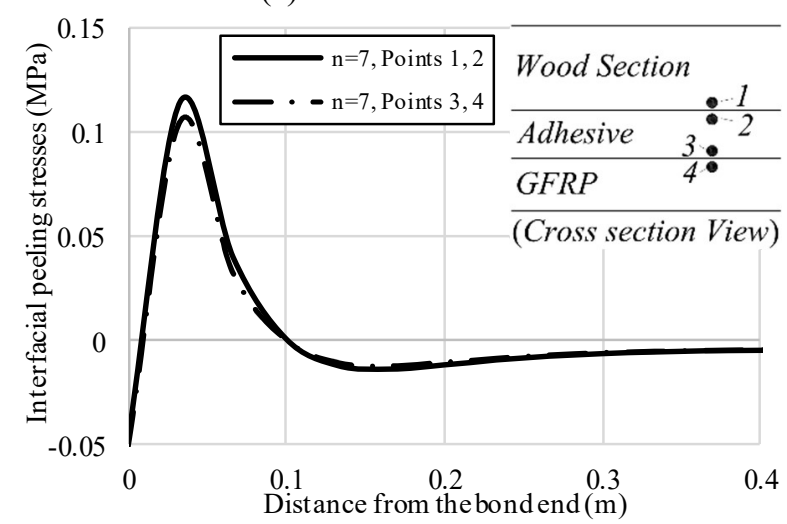

(c) Present solution

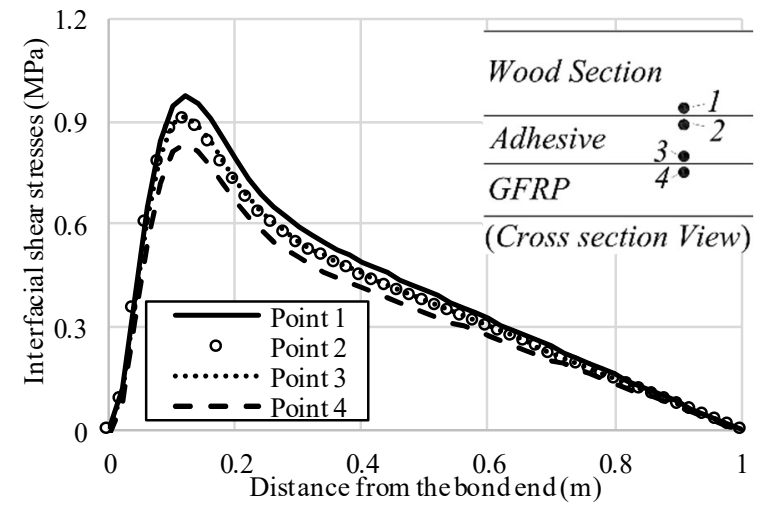

(b) 3D FEA (Mesh 2)

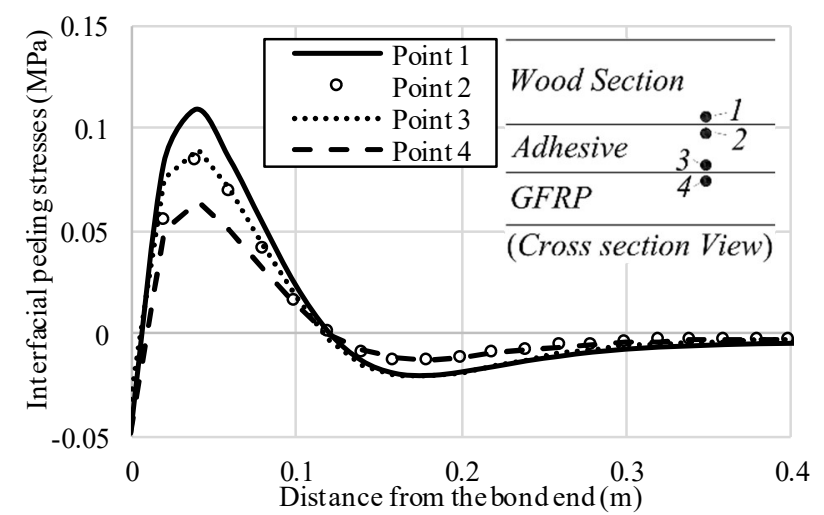

(d) 3D FEA (Mesh 2)

Figure 13. Interfacial stresses extracted at Points 1 to 4 from the present solution with $n=7$ and 3D FEA with Mesh 2 (a) Interfacial shear stresses- Present solution, (b) Interfacial shear stresses - 3D FEA, (c) Interfacial peeling stresses - Present solution, (d) Interfacial peeling stresses -3D FEA.

In addition to the case $\mathrm{L} / \mathrm{h}=10$, the interfacial stresses were investigated for two span-to-depth ratios $\mathrm{L} / \mathrm{h}=5$ and 20 . In both cases, Figure 14 shows very good agreement between the predicted shear and peeling stress distributions for $n=7$ and the 3D FEA solution. The peak shear stress predicted by the present solution are marginally smaller than those predicted by the 3D FEA. 


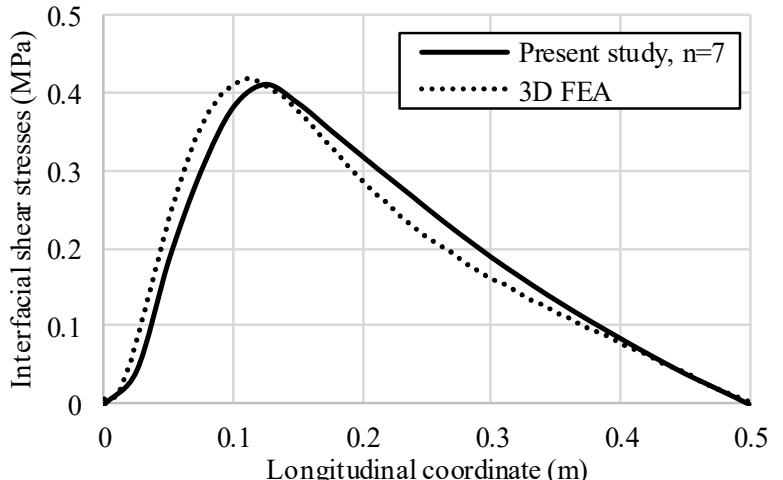

(a) $\mathrm{L} / \mathrm{h}=5$

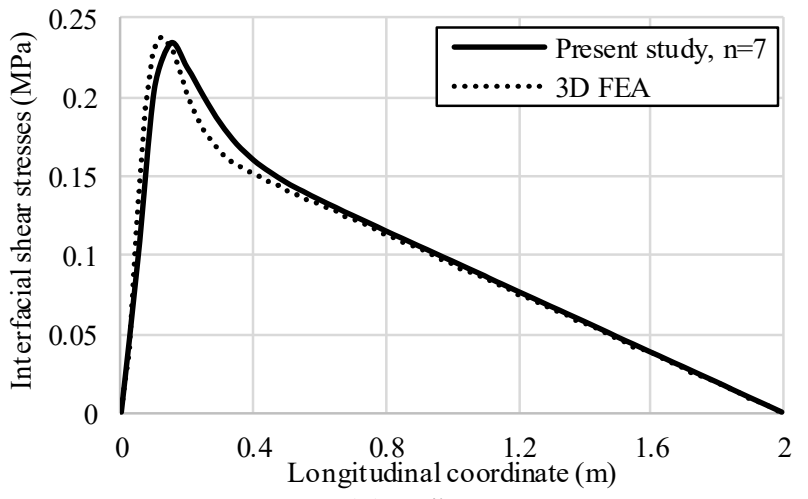

(c) $\mathrm{L} / \mathrm{h}=20$



(b) $\mathrm{L} / \mathrm{h}=5$

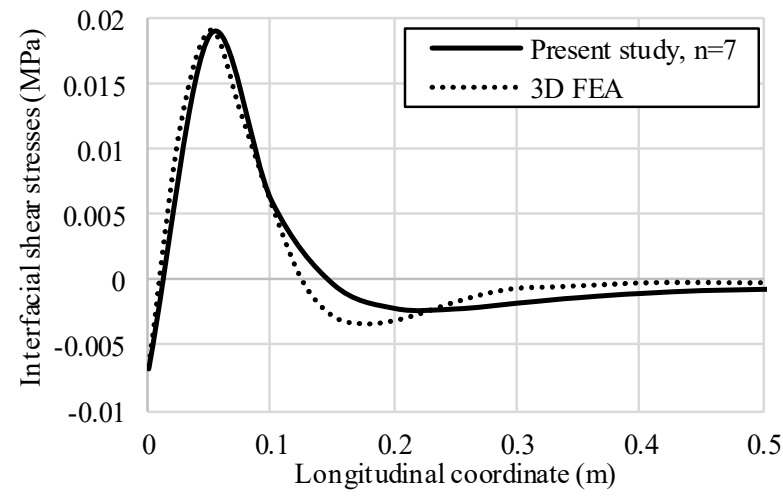

(d) $\mathrm{L} / \mathrm{h}=20$

Figure 14. Interfacial shear and peeling stresses for a wood beam bonded with a GFRP plate

Deflection of the composite system: The deflection for the case $\mathrm{L} / \mathrm{h}=10$ is determined based on the present solution, a modified Euler-Bernoulli beam (EB) solution with a transformed section treatment, and the 3D FEA. Unlike examples 1 and 2, the application of the Timoshenko solution is not possible in the absence of a shear modification factor for the composite section. The deflections predicted by the present theory with $n=3$ and $n=5$ were found to coincide with those of the 3D FEA, as all three solutions predicted a mid-span deflection of $9.9 \mathrm{~mm}$, suggesting that convergence is achieved for $n=3$. This value is $13.1 \%$ higher than that based on the EB solution. The difference is primarily attributed to shear deformation effects.

Longitudinal stress profile: Figure 15 provides a comparison for the longitudinal stress profile at mid-span as predicted by the present and 3D FEA solutions. In contrast to interfacial shear and peeling stresses which converged for $n=7$, longitudinal stresses in the wood beam, adhesive, and GFRP are observed to converge for $n=3$. Excellent agreement is obtained between the present solution and the 3D FEA for the stresses in the wood, adhesive and GFRP. 


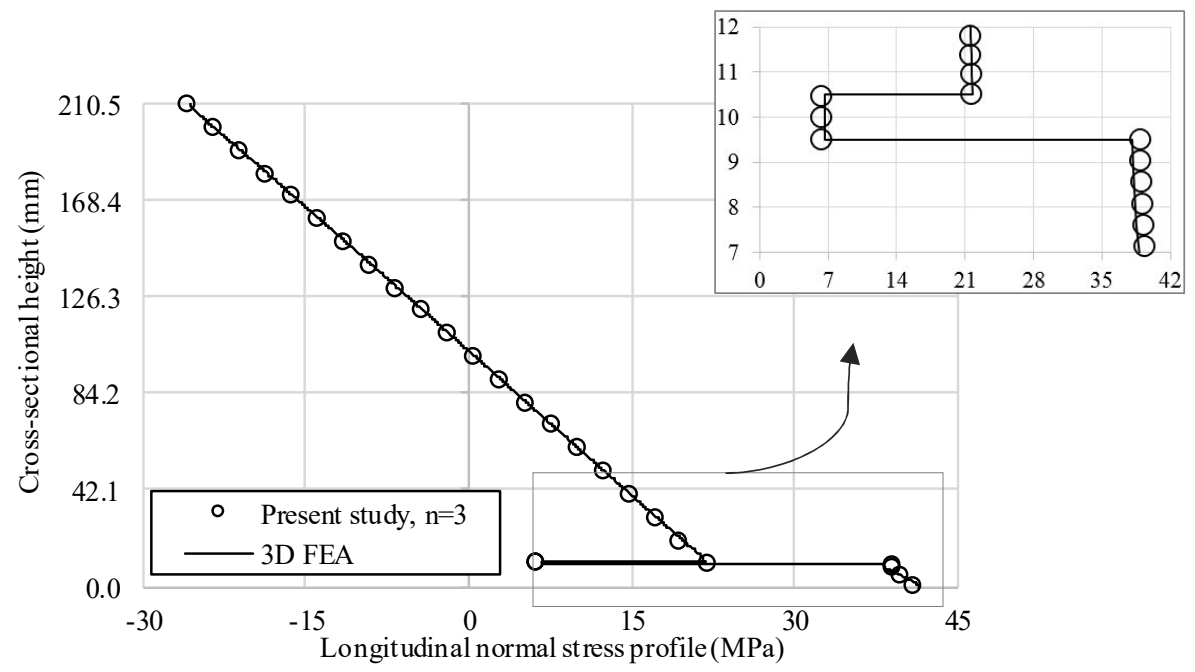

Figure 15. Longitudinal normal stresses between 3D FEA and present solution

\section{Example 4: Application to simply supported sandwich beam under line load}

A standard Hexlite 220 panel [29] is considered. The face skins are made of $0.5 \mathrm{~mm}$ thick Alumium $5251 \mathrm{H} 24$ with a Young modulus of $70 \mathrm{GPa}$ and a Poisson's ratio of 0.33 . The core is $25.4 \mathrm{~mm}$ thick with a Young modulus of $1.0 \mathrm{GPa}$, and a shear modulus of $220 \mathrm{MPa}$. Cross-section width is $0.5 \mathrm{~m}$ and beam span is $2.0 \mathrm{~m}$. The beam is simply supported at both ends (Figure 16) and subjected to a mid-span line load $P=313 N$ along the section width.

Because stresses are highly concentrated near mid-span where the load is applied, the present finite elements adopt more elements near mid-span (Figure 17a). A convergence study for the stresses showed that a total 30 elements are required for the central $100 \mathrm{~mm}$-long segment while only 9 elements are needed for the $900 \mathrm{~mm}$-long end segments. The mid-span point load $P=312 N$ is applied to the left node of element No. 25 depicted in Figure 17a and at height $y=-h / 2$ of section $z=0$ of the element. The body forces corresponding to the point load are expressed as $p_{y}=p_{y}(y, z)=P \operatorname{Dirac}(z) \operatorname{Dirac}\left(y+h_{1}\right), p_{z}=0$. Substitution into Eq. (4b) yields $\bar{\tau}(y, z)=0$, and $\bar{\sigma}_{y}(y, z)=P \operatorname{Dirac}(z)$. Further substitution into Eq. (A.46a-b) yields $\rho_{1}(z)$ and $\rho_{2}(z)$, and subsequent substitution into Eq. (9a-c) yields $\sigma_{z}^{*}(y, z)=\left[y^{n-2} \rho_{1}(z)+y^{n-1} \rho_{2}(z)\right] P z$, $\sigma_{y}^{*}(y, z)=P \operatorname{Dirac}(z)\left[1+\beta_{n-1}(y) \rho_{1}(z)+\beta_{n}(y) \rho_{2}(z)\right], \quad \tau^{*}(y, z)=-\left[\alpha_{n-1}(y) \rho_{1}(z)+\alpha_{n}(y) \rho_{2}(z)\right] P$. The ABAQUS 3D FEA solution is meshed in a manner similar to Example 3. In the 3D model, four elements are taken across the thicknesses of the two faces, 40 elements across the core depth, 
120 elements across the section width, and 600 elements along the span. The point load is applied by using a line load across the beam width to avoid the localization of stresses. The pin supports are modelled by applying the multi-point constrain *MPC, type BEAM in the Abaqus model. Deflections and longitudinal stresses: Figure $17 \mathrm{~b}$ presents the deflection curve predicted by the present solution with $\mathrm{n}=3,5$ and the $3 \mathrm{D}$ FEA solution. All solutions predict a nearly identical midspan deflection of $8.0 \mathrm{~mm}$. Figure 18a,b show the distribution of the longitudinal normal stresses at the bottom fiber of the bottom face and the bottom fiber of the core along the $\mathrm{z}$ coordinate as predicted based on the present solution and the 3D FEA solution. The results are also observed to essentially coincide.

Interfacial shear and peeling stresses: The shear stress distributions predicted by both solutions (Figure 19a) is anti-symmetric about the mid-span point and exhibit larger concentrations near the point of load application. Shear stresses are found to peak at mid-span in a manner similar to Pappada et al. [30]. The predicted interfacial normal stresses at the top interface are symmetric about the mid-span (Figure 19b). The fact that both interfacial shear and peeling stresses peak at mid-span is consistent with the shear failure mode of the core or face delamination mode, both reported in the experiments of Pappada et al. [30]. Figure 19c-d show that the shear and peeling stresses at the bottom interface predicted by both models are in close agreement.

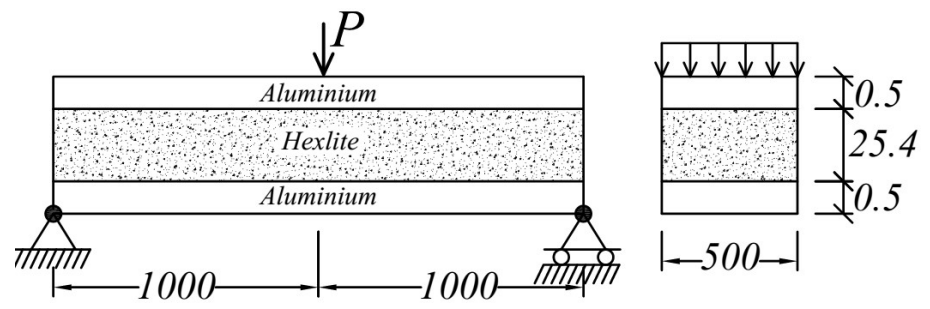

Figure 16. A sandwich beam with softcore under a mid-span point load

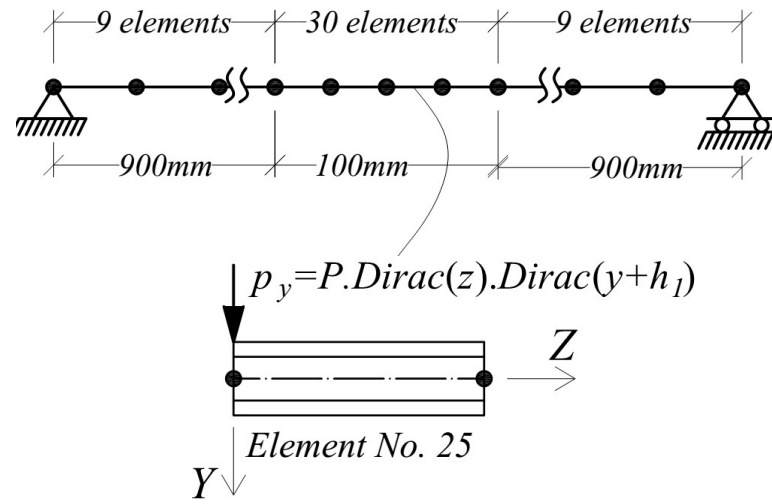

(a)

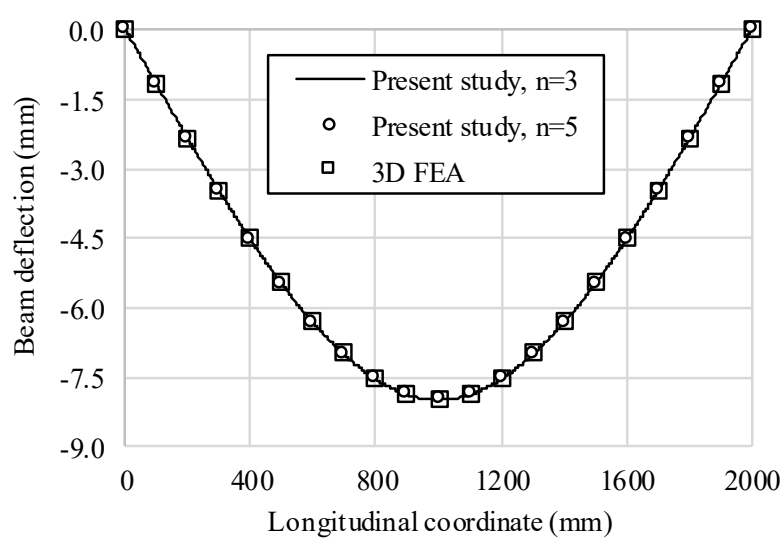

(b)

Figure 17. (a) Mesh adopted in present solution and loading and (b) Deflections 




(a)



(b)

Figure 18. Longitudinal normal stresses for sandwich beam with a soft core at (a) bottom fiber of the bottom face, and (b) bottom fibre of the core

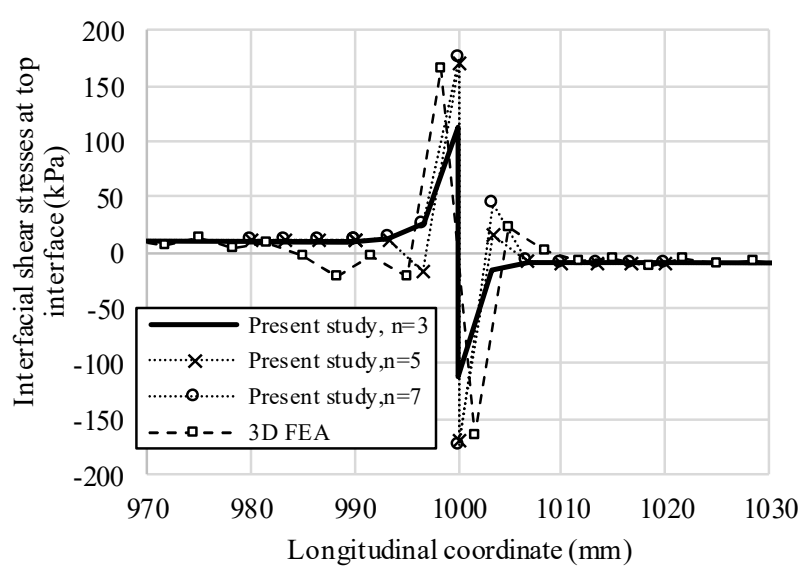

(a)

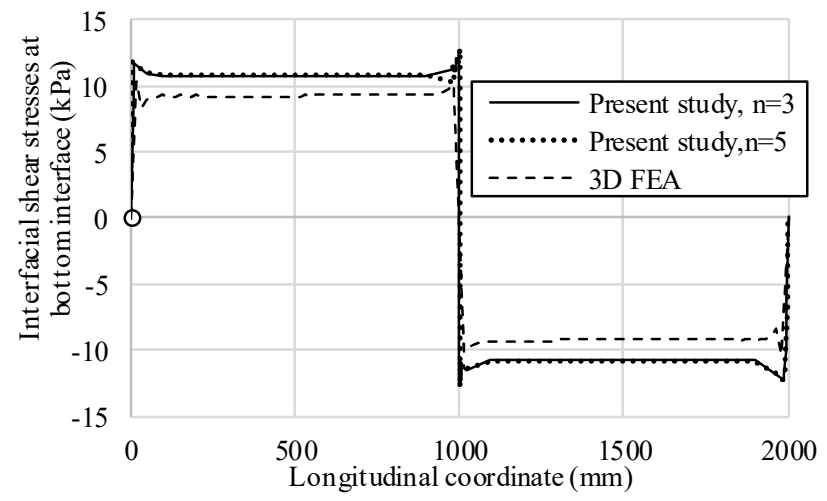

(c)

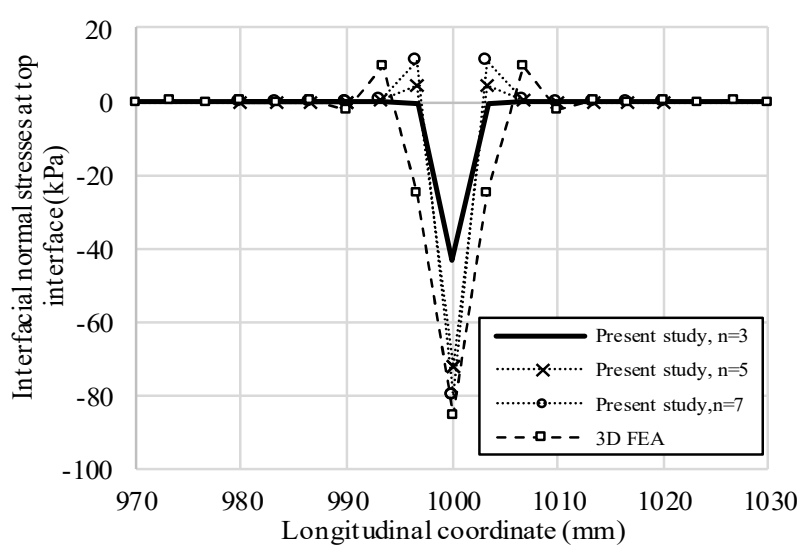

(b)

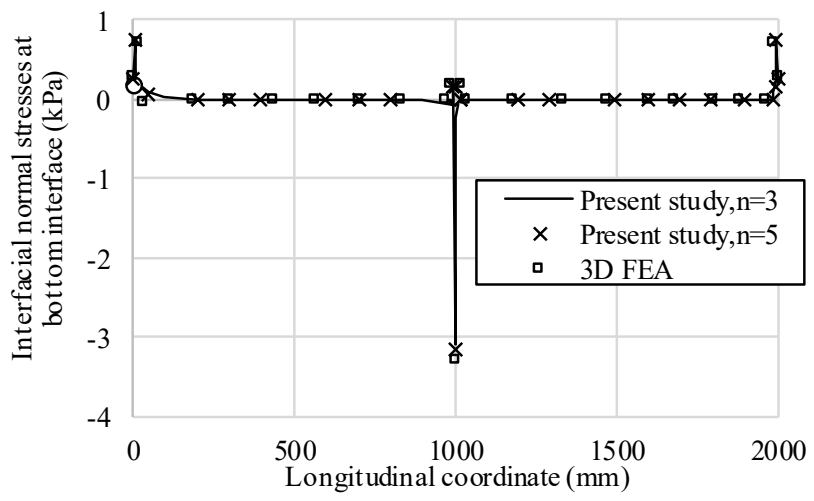

(d)

Figure 19. Interfacial shear and peeling stresses of sandwich beam: (a) shear stresses at top interface, (b) peeling stresses at top interface, (c) shear stresses at bottom interface, (d) peeling stresses at bottom interface.

\section{Example 5 - Application to wide flange steel beam strengthened with GFRP plate}

A W150x13 steel beam $($ depth $=148 \mathrm{~mm}$, flange width $=100 \mathrm{~mm}$, flange thickness $=4.9 \mathrm{~mm}$, and web thickness $=4.3 \mathrm{~mm}$ ) is strengthened with a $100 \mathrm{~mm}$ wide $\times 19 \mathrm{~mm}$-thick GFRP plate through a 1-mm thick adhesive layer (Figure 20). Moduli of elasticity for steel, GFRP, and adhesive are 
respectively 200, 17.2, and 3.18 GPa, and Poisson's ratios are taken as 0.3 for all materials. Two spans $\mathrm{L}=1.5 \mathrm{~m}$ and $3.0 \mathrm{~m}$ are considered. The steel beam is supported by two cleat angles which can be idealized as pin supports. A downward traction $\sigma=0.43 M P a$ is applied to at top face for the $1.5 \mathrm{~m}$ span while $\sigma=0.215 \mathrm{MPa}$ is applied for the $3.0 \mathrm{~m}$ span (in order to ensure that peak deflections remain within allowable limits). It is required to compare the mid-span deflection, the longitudinal normal stress profile at mid-span and the interfacial shear and peeling stresses as predicted by the 3D FEA and the present solution.

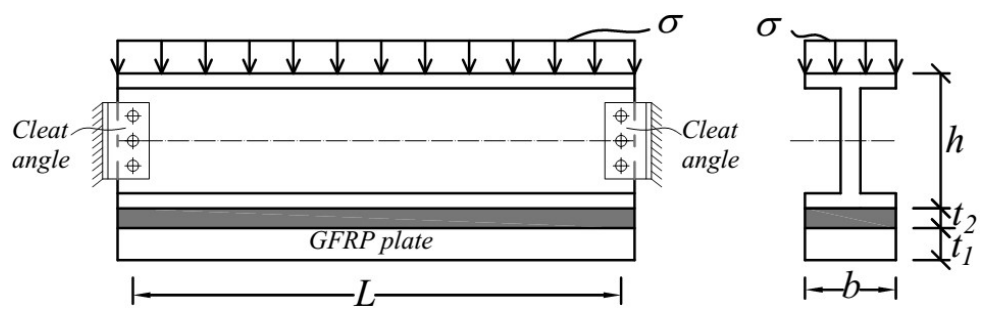

Figure 20. Beam elevation and cross-section

Figure 21a-b show good agreement between the longitudinal stress profiles predicted by the present theory with $n=3$ and the 3D FEA. When higher order stress terms are taken (i.e., $n=5,7)$, the predicted stress profiles are found to nearly coincide with those based on the 3D FEA solution. Figure 22a-d present the interfacial stresses obtained from the present solutions with $\mathrm{n}=3,5,7$ and the 3D FEA solutions for spans $\mathrm{L}=1.5 \mathrm{~m}$ and $\mathrm{L}=3.0 \mathrm{~m}$, respectively. The stresses in the 3D FEA solution are the average values across the cross-sectional width. The stresses predicted by the present solution with $n=3$ are found smaller than those based on $n=5$ or 7 while the predictions of solutions with $n=5$ and 7 are nearly identical to the 3D FEA predictions.

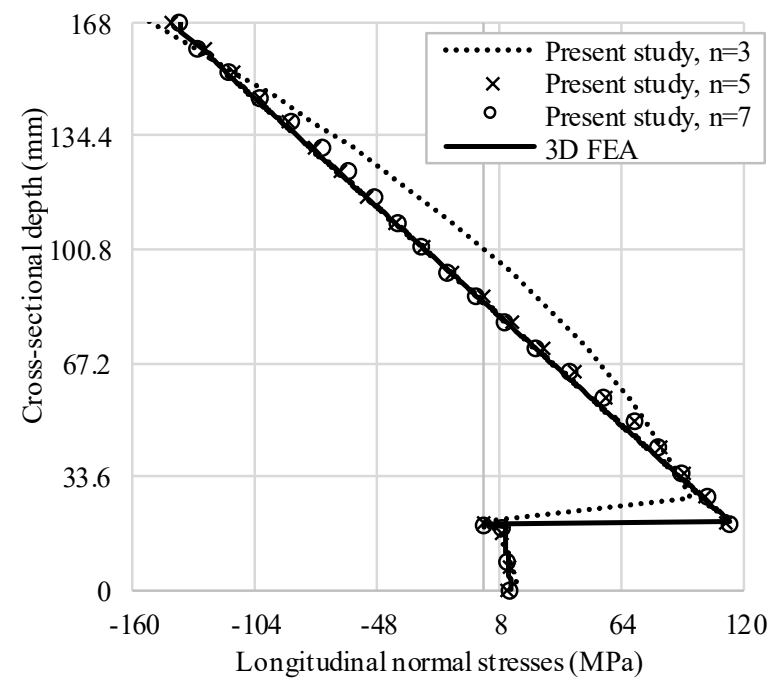

(a) $\mathrm{L}=1.5 \mathrm{~m}$

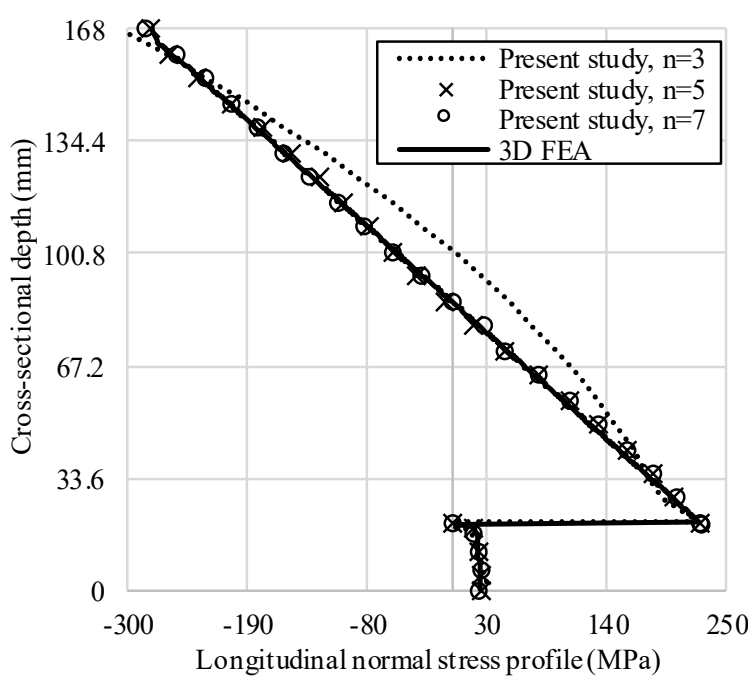

(b) $\mathrm{L}=3.0 \mathrm{~m}$

Figure 21. Longitudinal normal stress profiles at mid-span cross-section 


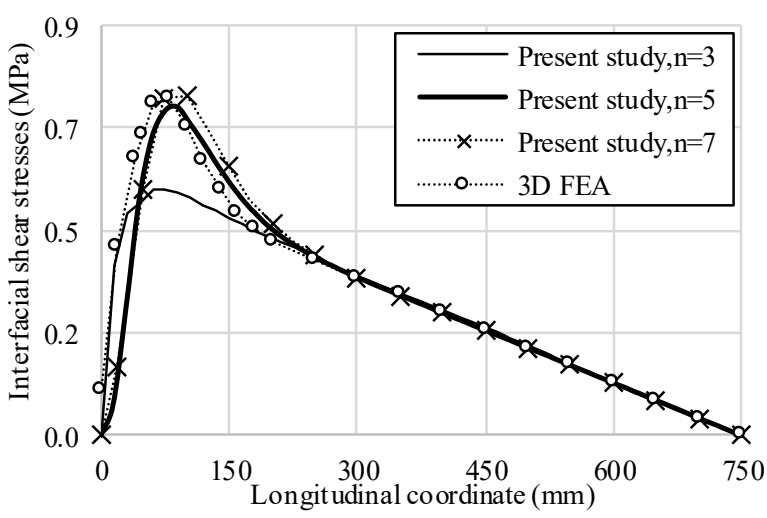

(a) $\mathrm{L}=1.5 \mathrm{~m}$

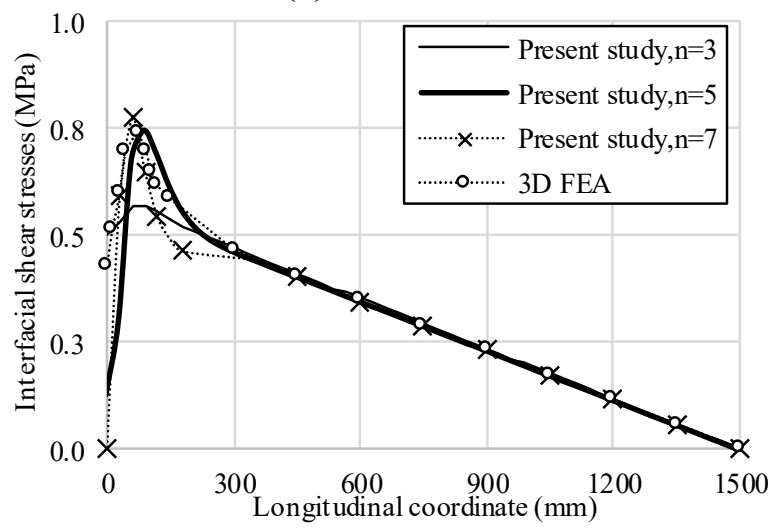

(c) $\mathrm{L}=3.0 \mathrm{~m}$



(b) $\mathrm{L}=1.5 \mathrm{~m}$



(d) $\mathrm{L}=3.0 \mathrm{~m}$

Figure 22. Comparisons of the interfacial shear and peeling stresses obtained from the present solution and the 3D FEA solutions

\section{Example 6-Sandwich beam with layers of equal thicknesses}

A sandwich beam has three layers, each having a $50 \mathrm{~mm}$ thickness. Beam width is $80 \mathrm{~mm}$. The span $L$ is taken as $600 \mathrm{~mm}$ and $1500 \mathrm{~mm}$. The beam is subjected to a transverse uniform traction $\sigma\left(-h_{1}, z\right)=0.1 M P a$ acting on the top face while other tractions vanish, i.e., $\tau\left(-h_{1}, z\right)=$ $\sigma\left(h_{2}, z\right)=\tau\left(h_{2}, z\right)=0$. Material properties of the faces are $E_{z}=10, E_{x}=E_{y}=4, G_{x y}=G_{x z}=$ $G_{y z}=0.8 G P a$, and $\mu_{y z}=\mu_{x y}=\mu_{x z}=0.25$ while the core material properties are $E_{z}=E_{x}=E_{y}=2 \mathrm{GPa}, G_{x y}=G_{x z}=G_{y z}=0.24 \mathrm{GPa}$, and $\mu_{y z}=\mu_{x y}=\mu_{x z}=0.25$ [31]. Figures 23a$\mathrm{b}$ show the longitudinal normal stress profiles at mid-span as predicted from the present solution with $n=3$. The excellent agreement observed with the 3D FEA stress profiles suggests the present solution is able to accurately capture the zigzag stress profiles. 


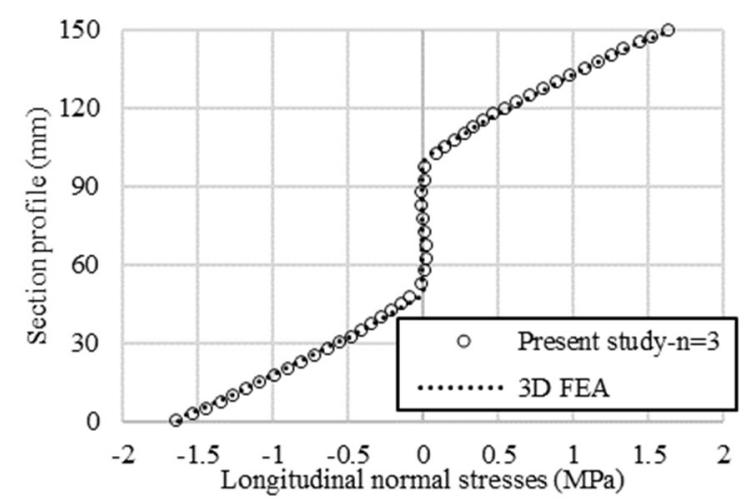

(a) span $\mathrm{L}=600 \mathrm{~mm}$



(b) span $\mathrm{L}=1500 \mathrm{~mm}$

Figure 23. Longitudinal normal stress profile at midspan for a sandwich beam with equal thickness layers

\section{Summary and Conclusions}

The present study has successfully developed a complementary strain energy variational principle for the analysis of homogeneous and multilayered beams. A finite element formulation was then developed based on the variational principle. The accuracy of the predictions of the present solution was shown to increase with the number of stress terms taken. Comparisons with other beam theories, elasticity theory solution and 3D FEA solutions suggest the validity of the present formulation for homogeneous and layered beams. The main findings of the studies are:

(1) The present solution captures shear deformation effects in a natural way that does not require the introduction of shear modification factors.

(2) The solution converges to the deflections from above in contrast to conventional solutions which tend to converge to the deflections from below.

(3) The present solution captures the nonlinear distribution of the longitudinal normal stress across the cross-section depth for short-span beams. Also, it captures the nonlinear distribution of the transverse normal stresses across the section depth.

(4) Unlike conventional 3D FEA solutions that interpolate the displacement fields, which violate the local equilibrium condition at the interfaces, the present solution satisfies the equilibrium condition at the interface in an exact sense as illustrated in Example 3.

(5) The high interfacial shear and peeling stresses predicted by the present solution agree well with the 3D FEA solution. Examples 3 through 5 suggest that the present solution with $n=7$ provides reliable predictions for the interfacial stresses.

(6) The computational effort involved in the present solution is orders of magnitudes less than that of 3D FEA analysis, particularly for the analysis of layered beams. The present solutions, 
implemented in non-compiled MATLAB script files, took between 3-15 minutes to conduct the runs in Examples 3-5 on a computer with two Intel(R) Xeon(R) CPU E5-24300 processors at 2.20 GHz speed, and 64.0 GB memory RAM. In comparison, the 3D FEA under ABAQUS took 3.8 - 6.9 hours per run on the same computer. The new solution also involves less effort in modelling and post-processing compared to 3D FEA solutions.

\section{Acknowledgements}

The authors gratefully acknowledge scholarship supports from the University of Ottawa and Ontario Graduate Scholarship (OGS) to the first author and research funding from the Natural Sciences and Engineering Research Council (NSERC) of Canada to the second and third authors.

\section{Appendix A -Developing Expressions for Stress Fields}

Starting with Eqs. (1) and (3a-b), the present appendix provides the details for obtaining Eq. (6). The first step (Section A.1) is to reduce the number of unknown functions $F_{i}(z)$ and the second step is to express stresses in terms of stress resultant (Section A.2)

\section{A.1. Reducing the Number of Unknown Functions $F_{i}(z)$}

From Eqs. (3a-b), by setting $y=h_{2}$, the stresses at the bottom fiber (Fig. 2) are obtained as

$$
\begin{aligned}
\tau\left(h_{2}, z\right) & =\bar{\tau}\left(h_{2}, z\right)-\sum_{i=1}^{n} \alpha_{i}\left(h_{2}\right) F_{i}^{\prime}(z)-\sum_{k=1}^{p-1} a_{k}\left(h_{2}\right) J_{k}^{\prime}(z) \\
\sigma_{y}\left(h_{2}, z\right) & =\bar{\sigma}_{y}\left(h_{2}, z\right)+\sum_{i=1}^{n} \beta_{i}\left(h_{2}\right) F_{i}^{\prime \prime}(z)+\sum_{k=1}^{p-1} c_{k}\left(h_{2}\right) J_{k}^{\prime \prime}(z)
\end{aligned}
$$

From Equations (A.43a-b), by expressing functions $F_{n-1}$ and $F_{n}$ in terms of remaining functions $F_{i}(z)(i=1, \ldots, n-2)$, one obtains

$$
\begin{aligned}
& \alpha_{n-1}\left(h_{2}\right) F_{n-1}{ }^{\prime}(z)+\alpha_{n}\left(h_{2}\right) F_{n}^{\prime}(z)=\bar{\tau}\left(h_{2}, z\right)-\tau\left(h_{2}, z\right)-\sum_{i=1}^{n-2} \alpha_{i}\left(h_{2}\right) F_{i}^{\prime}(z)-\sum_{k=1}^{p-1} a_{k}\left(h_{2}\right) J_{k}{ }^{\prime}(z) \\
& \beta_{n-1}\left(h_{2}\right) F_{n-1}^{\prime \prime}(z)+\beta_{n}\left(h_{2}\right) F_{n}^{\prime \prime}(z)=\sigma_{y}\left(h_{2}, z\right)-\bar{\sigma}_{y}\left(h_{2}, z\right)-\sum_{i=1}^{n-2} \beta_{i}\left(h_{2}\right) F_{i}^{\prime \prime}(z)-\sum_{k=1}^{p-1} c_{k}\left(h_{2}\right) J_{k}^{\prime \prime}(z)
\end{aligned}
$$

From Eq. (A.44a), by differentiation with respect to $z$, combining with Eq. (A.44b) and solving for $F_{n-1}^{\prime \prime}$ and $F_{n}^{\prime \prime}$, one obtains

$$
F_{n-1}^{\prime \prime}(z)=\rho_{1}(z)-\sum_{i=1}^{n-2} \lambda_{1 i} F_{i}^{\prime \prime}(z)-\sum_{k=1}^{p-1} \xi_{1 k} J_{k}^{\prime \prime}(z) ; \quad F_{n}^{\prime \prime}(z)=\rho_{2}(z)-\sum_{i=1}^{n-2} \lambda_{2 i} F_{i}^{\prime \prime}(z)-\sum_{k=1}^{p-1} \xi_{2 k} J_{k}^{\prime \prime}(z) \quad \text { (A. } 45 \mathrm{a}-
$$

b)

where the following functions and constants have been defined: 


$$
\begin{aligned}
& \rho_{1}(z)=\frac{\alpha_{n}\left(h_{2}\right)\left[\sigma_{y}\left(h_{2}, z\right)-\bar{\sigma}_{y}\left(h_{2}, z\right)\right]-\beta_{n}\left(h_{2}\right)\left[\bar{\tau}^{\prime}\left(h_{2}, z\right)-\tau^{\prime}\left(h_{2}, z\right)\right]}{\alpha_{n}\left(h_{2}\right) \beta_{n-1}\left(h_{2}\right)-\alpha_{n-1}\left(h_{2}\right) \beta_{n}\left(h_{2}\right)} ; \\
& \rho_{2}(z)=-\frac{\alpha_{n-1}\left(h_{2}\right)\left[\sigma_{y}\left(h_{2}, z\right)-\bar{\sigma}_{y}\left(h_{2}, z\right)\right]-\beta_{n-1}\left(h_{2}\right)\left[\bar{\tau}^{\prime}\left(h_{2}, z\right)-\tau^{\prime}\left(h_{2}, z\right)\right]}{\alpha_{n}\left(h_{2}\right) \beta_{n-1}\left(h_{2}\right)-\alpha_{n-1}\left(h_{2}\right) \beta_{n}\left(h_{2}\right)} ; \\
& \lambda_{1 i}=\frac{\alpha_{n}\left(h_{2}\right) \beta_{i}\left(h_{2}\right)-\beta_{n}\left(h_{2}\right) \alpha_{i}\left(h_{2}\right)}{\alpha_{n}\left(h_{2}\right) \beta_{n-1}\left(h_{2}\right)-\alpha_{n-1}\left(h_{2}\right) \beta_{n}\left(h_{2}\right)} ; \quad \lambda_{2 i}=-\frac{\alpha_{n-1}\left(h_{2}\right) \beta_{i}\left(h_{2}\right)-\beta_{n-1}\left(h_{2}\right) \alpha_{i}\left(h_{2}\right)}{\alpha_{n}\left(h_{2}\right) \beta_{n-1}\left(h_{2}\right)-\alpha_{n-1}\left(h_{2}\right) \beta_{n}\left(h_{2}\right)} ; \\
& \xi_{1 k}=\frac{\alpha_{n}\left(h_{2}\right) c_{k}\left(h_{2}\right)-\beta_{n}\left(h_{2}\right) a_{k}\left(h_{2}\right)}{\alpha_{n}\left(h_{2}\right) \beta_{n-1}\left(h_{2}\right)-\alpha_{n-1}\left(h_{2}\right) \beta_{n}\left(h_{2}\right)} ; \quad \xi_{2 k}=-\frac{\alpha_{n-1}\left(h_{2}\right) c_{k}\left(h_{2}\right)-\beta_{n-1}\left(h_{2}\right) a_{k}\left(h_{2}\right)}{\alpha_{n}\left(h_{2}\right) \beta_{n-1}\left(h_{2}\right)-\alpha_{n-1}\left(h_{2}\right) \beta_{n}\left(h_{2}\right)} ;
\end{aligned}
$$

f)

Traction equilibrium requires the stress fields $\sigma_{y}(y, z)$ and $\tau(y, z)$ at the faces $z=-h_{1}$ and $z=h_{2}$ to be equal to the externally applied surface tractions. By integrating Eqs. (A.45a-b) twice from 0 to $z$, functions $F_{n-1}(z)$ and $F_{n}(z)$ are obtained as

$$
\begin{aligned}
F_{n-1}(z)= & \int_{0}^{z} \int_{0}^{z} \rho_{11}(z) d z-\sum_{i=1}^{n-2} \lambda_{1 i} F_{i}(z)+\sum_{i=1}^{n-2} \lambda_{1 i} F_{i}(0)+z \sum_{i=1}^{n-2} \lambda_{1 i} F_{i}^{\prime}(0)+F_{n-1}(0)+z F_{n-1}^{\prime}(0) \\
& -\sum_{k=1}^{p-1} \xi_{1 k} J_{k}(z)+\sum_{k=1}^{p-1} \xi_{1 k} J_{k}(0)+z \sum_{k=1}^{p-1} \xi_{1 k} J_{k}^{\prime}(0) \\
F_{n}(z)= & \int_{0}^{z} \int_{0}^{z} \rho_{21}(z) d z d z-\sum_{i=1}^{n-2} \lambda_{2 i} F_{i}(z)+\sum_{i=1}^{n-2} \lambda_{2 i} F_{i}(0)+z \sum_{i=1}^{n-2} \lambda_{2 i} F_{i}^{\prime}(0)+F_{n}(0)+z F_{n}^{\prime}(0) \\
& -\sum_{k=1}^{p-1} \xi_{2 k} J_{k}(z)+\sum_{k=1}^{p-1} \xi_{2 k} J_{k}(0)+z \sum_{k=1}^{p-1} \xi_{2 k} J_{k}^{\prime}(0)
\end{aligned}
$$

From Eqs. (A.47a-b), by substituting into Eqs. (1) and (3a-b), one obtains 


$$
\begin{aligned}
\sigma_{z}(y, z)= & \int_{0}^{z} \int_{0}^{z}\left(y^{n-2} \rho_{1}(z)+y^{n-1} \rho_{2}(z)\right) d z d z+\left(y^{n-2} F_{n-1}(0)+z y^{n-2} F_{n-1}^{\prime}(0)+y^{n-1} F_{n}(0)+z y^{n-1} F_{n}^{\prime}(0)\right) \\
& +\sum_{i=1}^{n-2}\left(y^{n-2} \lambda_{1 i}+y^{n-1} \lambda_{2 i}\right) F_{i}(0)+\sum_{i=1}^{n-2}\left(y^{n-2} \lambda_{1 i}+y^{n-1} \lambda_{2 i}\right) z F_{i}^{\prime}(0)-\sum_{i=1}^{n-2}\left(y^{n-2} \lambda_{1 i}+y^{n-1} \lambda_{2 i}-y^{i-1}\right) F_{i}(z) \\
& +\sum_{k=1}^{p-1}\left(y^{n-2} \xi_{1 k}+y^{n-1} \xi_{2 k}\right) J_{k}(0)+\sum_{k=1}^{p-1}\left(y^{n-2} \xi_{1 k}+y^{n-1} \xi_{2 k}\right) z J_{k}^{\prime}(0)-\sum_{k=1}^{p-1}\left[y^{n-2} \xi_{1 k}+y^{n-1} \xi_{2 k}-H\left(y-y_{k}\right)\right] J_{k}(z) \\
\tau(y, z)= & \bar{\tau}(y, z)-\int_{0}^{z}\left[\alpha_{n-1}(y) \rho_{1}(z)+\alpha_{n}(y) \rho_{2}(z)\right] d z-\alpha_{n-1}(y) F_{n-1}^{\prime}(0)-\alpha_{n}(y) F_{n}^{\prime}(0) \\
& -\sum_{i=1}^{n-2}\left[\alpha_{n-1}(y) \lambda_{1 i}+\alpha_{n}(y) \lambda_{2 i}\right] F_{i}^{\prime}(0)+\sum_{i=1}^{n-2}\left[\alpha_{n-1}(y) \lambda_{1 i}+\alpha_{n}(y) \lambda_{2 i}-\alpha_{i}(y)\right] F_{i}^{\prime}(z) \\
& -\sum_{k=1}^{p-1}\left[\alpha_{n-1}(y) \xi_{1 k}+\alpha_{n}(y) \xi_{2 k}\right] J_{k}^{\prime}(0)+\sum_{k=1}^{p-1}\left[\alpha_{n-1}(y) \xi_{1 k}+\alpha_{n}(y) \xi_{2 k}-a_{k}(y)\right] J_{k}^{\prime}(z) \\
\sigma_{y}(y, z)= & \sigma_{y}(y, z)+\beta_{n-1}(y) \rho_{1}(z)+\beta_{n}(y) \rho_{2}(z)+\sum_{i=1}^{n-2}\left[\beta_{i}(y)-\beta_{n-1}(y) \lambda_{1 i}-\beta_{n}(y) \lambda_{2 i}\right] F_{i}^{\prime \prime}(z) \\
& +\sum_{k=1}^{p-1}\left[c_{k}(y)-\beta_{n-1}(y) \xi_{1 k}-\beta_{n}(y) \xi_{2 k}\right] J_{k}^{\prime \prime}(z)
\end{aligned}
$$

c)

\section{A.2. Expressing Stress Constants in terms of Stress Resultants}

Constants $F_{n-1}(0), F_{n-1}^{\prime}(0), F_{n}(0)$ and $F_{n}^{\prime}(0)$ appearing in Eqs. (A.48a-b) are then eliminated by using four equations: the first one is obtained by differentiating Eqs. (A.48a-b) with respect to $z$, and substituting $F_{n-1}^{\prime}$ and $F_{n}^{\prime}$ into Eq. (A.44a), yielding

$$
\begin{aligned}
& \left\{\alpha_{n-1}\left(h_{2}\right) \int_{0}^{z} \rho_{1}(z) d z+\alpha_{n}\left(h_{2}\right) \int_{0}^{z} \rho_{2}(z) d z-\left[\bar{\tau}\left(h_{2}, z\right)-\tau\left(h_{2}, z\right)\right]\right\}+ \\
& +\left\{-\alpha_{n-1}\left(h_{2}\right) \sum_{i=1}^{n-2} \lambda_{1 i} F_{i}^{\prime}(z)-\alpha_{n}\left(h_{2}\right) \sum_{i=1}^{n-2} \lambda_{2 i} F_{i}^{\prime}(z)+\sum_{i=1}^{n-2} \alpha_{i}\left(h_{2}\right) F_{i}^{\prime}(z)\right\}+ \\
& +\left\{-\alpha_{n-1}\left(h_{2}\right) \sum_{k=1}^{p-1} \xi_{1 k} J_{k}^{\prime}(z)-\alpha_{n}\left(h_{2}\right) \sum_{k=1}^{p-1} \xi_{2 k} J_{k}^{\prime}(z)+\sum_{k=1}^{p-1} a_{k}\left(h_{2}\right) J_{k}{ }^{\prime}(z)\right\}+ \\
& +\alpha_{n-1}\left(h_{2}\right) \sum_{k=1}^{p-1} \xi_{1 k} J_{k}^{\prime}(0)+\alpha_{n}\left(h_{2}\right) \sum_{k=1}^{p-1} \xi_{2 k} J_{k}^{\prime}(0)+\alpha_{n-1}\left(h_{2}\right) \sum_{i=1}^{n-2} \lambda_{1 i} F_{i}^{\prime}(0)+ \\
& +\alpha_{n}\left(h_{2}\right) \sum_{i=1}^{n-2} \lambda_{2 i} F_{i}^{\prime}(0)+\alpha_{n-1}\left(h_{2}\right) F_{n-1}^{\prime}(0)+\alpha_{n}\left(h_{2}\right) F_{n}^{\prime}(0)=0
\end{aligned}
$$

From Eqs. (A.46a-b), by substituting into Eq.(A.49), one obtains 


$$
\begin{aligned}
& -\bar{\tau}\left(h_{2}, 0\right)+\tau\left(h_{2}, 0\right)-\sum_{i=1}^{n-2}\left[\alpha_{n-1}\left(h_{2}\right) \lambda_{1 i}+\alpha_{n}\left(h_{2}\right) \lambda_{2 i}-\alpha_{i}\left(h_{2}\right)\right] F_{i}^{\prime}(z)+ \\
& -\sum_{k=1}^{p-1}\left[\alpha_{n-1}\left(h_{2}\right) \xi_{1 k}+\alpha_{n}\left(h_{2}\right) \xi_{2 k}-a_{k}\left(h_{2}\right)\right] J_{k}^{\prime}(z)+\alpha_{n-1}\left(h_{2}\right) F_{n-1}^{\prime}(0)+\alpha_{n}\left(h_{2}\right) F_{n}^{\prime}(0) \\
& =-\alpha_{n-1}\left(h_{2}\right) \sum_{i=1}^{n-2} \lambda_{1 i} F_{i}^{\prime}(0)-\alpha_{n}\left(h_{2}\right) \sum_{i=1}^{n-2} \lambda_{2 i} F_{i}^{\prime}(0)-\alpha_{n-1}\left(h_{2}\right) \sum_{k=1}^{p-1} \xi_{1 k} J_{k}^{\prime}(0)-\alpha_{n}\left(h_{2}\right) \sum_{k=1}^{p-1} \xi_{2 k} J_{k}^{\prime}(0)
\end{aligned}
$$

From Eq. (A.50), by noting the identities $\alpha_{n-1}\left(h_{2}\right) \lambda_{1 i}+\alpha_{n}\left(h_{2}\right) \lambda_{2 i}-\alpha_{i}\left(h_{2}\right)=0$ and $\alpha_{n-1}\left(h_{2}\right) \xi_{1 k}+\alpha_{n}\left(h_{2}\right) \xi_{2 k}-a_{k}\left(h_{2}\right)=0$, one obtains

$\alpha_{n-1}\left(h_{2}\right) F_{n-1}^{\prime}(0)+\alpha_{n}\left(h_{2}\right) F_{n}^{\prime}(0)=\left[\bar{\tau}\left(h_{2}, 0\right)-\tau\left(h_{2}, 0\right)\right]-\sum_{i=1}^{n-2} \alpha_{i}\left(h_{2}\right) F_{i}^{\prime}(0)-\sum_{k=1}^{p-1} a_{k}\left(h_{2}\right) J_{k}^{\prime}(0)$

The other three equations are recovered by equating the stress resultants to internal forces $N(0), Q(0), M(0)$, i.e.,

$$
N(0)=\int_{A} \sigma_{z}(y, 0) d A ; \quad Q(0)=\int_{A} \tau(y, 0) d A ; \quad M(0)=\int_{A} y \sigma_{z}(y, 0) d A
$$

From Eqs. (1) and (3a-b), one can obtain the stress fields at $z=0$ as

$$
\begin{aligned}
\sigma_{z}(y, 0) & =y^{n-2} F_{n-1}(0)+y^{n-1} F_{n}(0)+\sum_{i=1}^{n-2} y^{i-1} F_{i}(0)+\sum_{k=1}^{p-1} H\left(y-y_{k}\right) J_{k}(z) \\
\tau(y, 0) & =\bar{\tau}(y, 0)-\alpha_{n-1}(y) F_{n-1}{ }^{\prime}(0)-\alpha_{n}(y) F_{n}{ }^{\prime}(0)-\sum_{i=1}^{n-2} \alpha_{i}(y) F_{i}{ }^{\prime}(0)-\sum_{k=1}^{p-1} a_{k}(y) J_{k}{ }^{\prime}(z)
\end{aligned}
$$

From Eqs. (A.53), by substituting into (A.52), one obtains

$$
\begin{aligned}
& \bar{\alpha}_{n-2} F_{n-1}(0)+\bar{\alpha}_{n-1} F_{n}(0)=N(0)-\sum_{i=1}^{n-2} \bar{\alpha}_{i-1} F_{i}(0)-\sum_{k=1}^{p-1} \int_{A} H\left(y-y_{k}\right) d A J_{k}(0) \\
& \hat{\alpha}_{n-1} F_{n-1}^{\prime}(0)+\widehat{\alpha}_{n} F_{n}^{\prime}(0)=-Q(0)+\int_{A} \bar{\tau}(y, 0) d A-\sum_{i=1}^{n-2} \widehat{\alpha}_{i} F_{i}^{\prime}(0)-\sum_{k=1}^{p-1} \int_{A} a_{k}(y) d A J_{k}^{\prime}(0) \\
& \bar{\alpha}_{n-1} F_{n-1}(0)+\bar{\alpha}_{n} F_{n}(0)=M(0)-\sum_{i=1}^{n-2} \bar{\alpha}_{i} F_{i}(0)-\sum_{k=1}^{p-1} \int_{A} y H\left(y-y_{k}\right) d A J_{k}(0)
\end{aligned}
$$

in which the sectional properties $\bar{\alpha}_{i}=\int_{A} y^{i} d A$ and $\hat{\alpha}_{i}=\int_{A} \alpha_{i}(y) d A$ have been defined. From Eq.(A.51 ) and Eqs. (A.54a-c), one recovers the following expressions for $F_{n-1}^{\prime}(0), F_{n}^{\prime}(0), F_{n-1}(0)$ and $F_{n}(0)$ 


$$
\begin{aligned}
& F_{n-1}^{\prime}(0)=\varsigma_{11}+\varsigma_{12} Q(0)+\sum_{i=1}^{n-2} \varsigma_{13 i} F_{i}^{\prime}(0)+\sum_{k=1}^{p-1} \varsigma_{14 k} J_{k}^{\prime}(0) \\
& F_{n}^{\prime}(0)=\varsigma_{21}+\varsigma_{22} Q(0)+\sum_{i=1}^{n-2} \varsigma_{23 i} F_{i}^{\prime}(0)+\sum_{k=1}^{p-1} \varsigma_{24 k} J_{k}^{\prime}(0) \\
& F_{n-1}(0)=\varsigma_{31} N(0)+\varsigma_{32} M(0)+\sum_{i=1}^{n-2} \varsigma_{33 i} F_{i}(0)+\sum_{k=1}^{p-1} \varsigma_{34 k} J_{k}(0) \\
& F_{n}(0)=\varsigma_{41} N(0)+\varsigma_{42} M(0)+\sum_{i=1}^{n-2} \varsigma_{43 i} F_{i}(0)+\sum_{k=1}^{p-1} \varsigma_{44 k} J_{k}(0)
\end{aligned}
$$

in which coefficients $\varsigma_{i j}$ depend on the cross-section geometry and are defined in Appendix B. By introducing the reduced number of terms $m=n-2$, and substituting from Eqs. (A.55a-d) into Eqs. (A.48a-c), one obtains Eq. (6).

\section{Appendix B- Procedure for developing the finite element formulation}

This appendix provides the steps for formulating the finite element solutions:

(1) Determine constants $\bar{\alpha}_{i}, \hat{\alpha}_{i}$ as defined in Eqs. (A.54):

$$
\bar{\alpha}_{i}=\int_{A} y^{i} d A, \quad \hat{\alpha}_{i}=\int_{A} \alpha_{i}(y) d A \quad(i=1,2, \ldots, m)
$$

(2) Starting from the definitions of $\alpha_{i}(y), \beta_{i}(y), c_{k}(y), a_{k}(y)$ as provided in Eqs. (5a-b), determine the following constants based on Eqs. (A.46c-f):

$$
\begin{array}{lll}
\lambda_{1 i}=\frac{\alpha_{n}\left(h_{2}\right) \beta_{i}\left(h_{2}\right)-\beta_{n}\left(h_{2}\right) \alpha_{i}\left(h_{2}\right)}{\alpha_{n}\left(h_{2}\right) \beta_{n-1}\left(h_{2}\right)-\alpha_{n-1}\left(h_{2}\right) \beta_{n}\left(h_{2}\right)} ; & \lambda_{2 i}=-\frac{\alpha_{n-1}\left(h_{2}\right) \beta_{i}\left(h_{2}\right)-\beta_{n-1}\left(h_{2}\right) \alpha_{i}\left(h_{2}\right)}{\alpha_{n}\left(h_{2}\right) \beta_{n-1}\left(h_{2}\right)-\alpha_{n-1}\left(h_{2}\right) \beta_{n}\left(h_{2}\right)} \\
\xi_{1 k}=\frac{\alpha_{n}\left(h_{2}\right) c_{k}\left(h_{2}\right)-\beta_{n}\left(h_{2}\right) a_{k}\left(h_{2}\right)}{\alpha_{n}\left(h_{2}\right) \beta_{n-1}\left(h_{2}\right)-\alpha_{n-1}\left(h_{2}\right) \beta_{n}\left(h_{2}\right)} ; & \xi_{2 k}=-\frac{\alpha_{n-1}\left(h_{2}\right) c_{k}\left(h_{2}\right)-\beta_{n-1}\left(h_{2}\right) a_{k}\left(h_{2}\right)}{\alpha_{n}\left(h_{2}\right) \beta_{n-1}\left(h_{2}\right)-\alpha_{n-1}\left(h_{2}\right) \beta_{n}\left(h_{2}\right)}
\end{array}
$$

(3) Given $\alpha_{i}(y)$ and $a_{k}(y)$ as given in Eq. (5a,c), $\bar{\alpha}_{i}$ and $\hat{\alpha}_{i}$ in Eqs. (B.56) and the knowing applied tractions, determine the following constants: 


$$
\begin{aligned}
& \varsigma_{11}=-\frac{\alpha_{n}\left(h_{2}\right) \int_{A} \bar{\tau}(y, 0) d A-\hat{\alpha}_{n}\left[\bar{\tau}\left(h_{2}, 0\right)-\tau\left(h_{2}, 0\right)\right]}{\hat{\alpha}_{n} \alpha_{n-1}\left(h_{2}\right)-\hat{\alpha}_{n-1} \alpha_{n}\left(h_{2}\right)} ; \quad \varsigma_{12}=\frac{\alpha_{n}\left(h_{2}\right)}{\hat{\alpha}_{n} \alpha_{n-1}\left(h_{2}\right)-\hat{\alpha}_{n-1} \alpha_{n}\left(h_{2}\right)} ; \\
& \varsigma_{13 i}=\frac{\alpha_{n}\left(h_{2}\right) \hat{\alpha}_{i}-\hat{\alpha}_{n} \alpha_{i}\left(h_{2}\right)}{\hat{\alpha}_{n} \alpha_{n-1}\left(h_{2}\right)-\hat{\alpha}_{n-1} \alpha_{n}\left(h_{2}\right)} ; \quad S_{14 k}=\frac{\alpha_{n}\left(h_{2}\right) \iint_{A} a_{k}(y) d A-\hat{\alpha}_{n} a_{k}\left(h_{2}\right)}{\hat{\alpha}_{n} \alpha_{n-1}\left(h_{2}\right)-\hat{\alpha}_{n-1} \alpha_{n}\left(h_{2}\right)} ; \\
& \varsigma_{21}=\frac{\alpha_{n-1}\left(h_{2}\right) \int_{A} \bar{\tau}(y, 0) d A-\hat{\alpha}_{n-1}\left[\bar{\tau}\left(h_{2}, 0\right)-\tau\left(h_{2}, 0\right)\right]}{\hat{\alpha}_{n} \alpha_{n-1}\left(h_{2}\right)-\hat{\alpha}_{n-1} \alpha_{n}\left(h_{2}\right)} ; \quad \varsigma_{22}=-\frac{\alpha_{n-1}\left(h_{2}\right)}{\hat{\alpha}_{n} \alpha_{n-1}\left(h_{2}\right)-\hat{\alpha}_{n-1} \alpha_{n}\left(h_{2}\right)} ; \\
& \varsigma_{23 i}=-\frac{\alpha_{n-1}\left(h_{2}\right) \hat{\alpha}_{i}-\hat{\alpha}_{n-1} \alpha_{i}\left(h_{2}\right)}{\hat{\alpha}_{n} \alpha_{n-1}\left(h_{2}\right)-\hat{\alpha}_{n-1} \alpha_{n}\left(h_{2}\right)} ; \quad \varsigma_{24 k}=-\frac{\alpha_{n-1}\left(h_{2}\right) \int_{A} a_{k}(y) d A-\hat{\alpha}_{n-1} a_{k}\left(h_{2}\right)}{\hat{\alpha}_{n} \alpha_{n-1}\left(h_{2}\right)-\hat{\alpha}_{n-1} \alpha_{n}\left(h_{2}\right)} ; \\
& \varsigma_{31}=\frac{\bar{\alpha}_{n}}{\bar{\alpha}_{n} \bar{\alpha}_{n-2}-\bar{\alpha}_{n-1} \bar{\alpha}_{n-1}} ; \quad \varsigma_{32}=-\frac{\bar{\alpha}_{n-1}}{\bar{\alpha}_{n} \bar{\alpha}_{n-2}-\bar{\alpha}_{n-1} \bar{\alpha}_{n-1}} ; \quad \varsigma_{33 i}=-\frac{\bar{\alpha}_{n} \bar{\alpha}_{i-1}-\bar{\alpha}_{n-1} \bar{\alpha}_{i}}{\bar{\alpha}_{n} \bar{\alpha}_{n-2}-\bar{\alpha}_{n-1} \bar{\alpha}_{n-1}} ; \\
& \varsigma_{34 k}=\frac{\bar{\alpha}_{n-1} \int_{A} y H\left(y-y_{k}\right) d A-\bar{\alpha}_{n} \int_{A} H\left(y-y_{k}\right) d A}{\bar{\alpha}_{n-2} \bar{\alpha}_{n}-\bar{\alpha}_{n-1} \bar{\alpha}_{n-1}} ; \varsigma_{44 k}=\frac{\bar{\alpha}_{n-1} \int_{A} H\left(y-y_{k}\right) d A-\bar{\alpha}_{n-2} \int_{A} y H\left(y-y_{k}\right) d A}{\bar{\alpha}_{n} \bar{\alpha}_{n-2}-\bar{\alpha}_{n-1} \bar{\alpha}_{n-1}} \\
& \varsigma_{41}=-\frac{\bar{\alpha}_{n-1}}{\bar{\alpha}_{n} \bar{\alpha}_{n-2}-\bar{\alpha}_{n-1} \bar{\alpha}_{n-1}} ; \quad \varsigma_{42}=\frac{\bar{\alpha}_{n-2}}{\bar{\alpha}_{n} \bar{\alpha}_{n-2}-\bar{\alpha}_{n-1} \bar{\alpha}_{n-1}} ; \quad \varsigma_{43 i}=\frac{\bar{\alpha}_{n-1} \bar{\alpha}_{i-1}-\bar{\alpha}_{n-2} \bar{\alpha}_{i}}{\bar{\alpha}_{n} \bar{\alpha}_{n-2}-\bar{\alpha}_{n-1} \bar{\alpha}_{n-1}} ;
\end{aligned}
$$

(4) Also, starting from the definitions of $\alpha_{i}(y), \beta_{i}(y), c_{k}(y), a_{k}(y)$ as provided in Eqs. (5ab) and the applied traction (i.e., $\left.\sigma_{y}\left(-h_{1}, z\right), \tau\left(-h_{1}, z\right), \sigma_{y}\left(h_{2}, z\right), \tau\left(h_{2}, z\right)\right)$, determine the following constants according to Eqs. (A.46a-b):

$$
\begin{aligned}
& \rho_{1}(z)=\left\{\alpha_{n}\left(h_{2}\right)\left[\sigma_{y}\left(h_{2}, z\right)-\bar{\sigma}_{y}\left(h_{2}, z\right)\right]-\beta_{n}\left(h_{2}\right)\left[\bar{\tau}^{\prime}\left(h_{2}, z\right)-\tau^{\prime}\left(h_{2}, z\right)\right]\right\} / \Lambda, \\
& \rho_{2}(z)=-\left\{\alpha_{n-1}\left(h_{2}\right)\left[\sigma_{y}\left(h_{2}, z\right)-\bar{\sigma}_{y}\left(h_{2}, z\right)\right]-\beta_{n-1}\left(h_{2}\right)\left[\bar{\tau}^{\prime}\left(h_{2}, z\right)-\tau^{\prime}\left(h_{2}, z\right)\right]\right\} / \Lambda \\
& \Lambda=\left\{\alpha_{n}\left(h_{2}\right) \beta_{n-1}\left(h_{2}\right)-\alpha_{n-1}\left(h_{2}\right) \beta_{n}\left(h_{2}\right)\right\}
\end{aligned}
$$

)

(5) Given $\varsigma_{i j}$ as given from Eq. (B.58), $\lambda_{i j}$ and $\xi_{i k}$ as given from Eq. (B.57), and $\alpha_{i}(y), \beta_{i}(y)$, $c_{k}(y), a_{k}(y)$ as given from Eqs. (5a), functions $a_{j i}(y), g_{j k}(y), b_{11}(y), b_{12}(y), b_{13}(y), b_{22}(y)($ $j=1,2, \ldots, 6)$ defined in Eq. (11) are determined.

(6) Given $\rho_{1}(z), \rho_{2}(z)$ from Eqs. (B.59), $\varsigma_{11}$ and $\varsigma_{21}$ from Eq. (B.58), and $\bar{\tau}(y, z), \bar{\sigma}_{y}(y, z)$ from Eq. (4), one recovers the expressions for $\sigma_{z}^{*}(y, z), \tau^{*}(y, z), \sigma_{y}^{*}(y, z)$ as defined in Eq.(9). 
(7) Given $\mathbf{a}_{1}(y), \mathbf{a}_{2}(y), \mathbf{a}_{3}(y, z), \mathbf{a}_{4}(y), \mathbf{a}_{\mathbf{5}}(y), \mathbf{a}_{\mathbf{6}}(y), \mathbf{b}_{\mathbf{1}}(y, z), \mathbf{b}_{\mathbf{2}}(y)$ as defined in Step 5, $\boldsymbol{\Lambda}_{\mathbf{F}}(z)$ as defined in Eq. (16), and $\Lambda_{\mathbf{R}}$ from Eq. (19), evaluate vectors $\left\{\mathbf{X}_{\sigma z}\right\},\left\{\mathbf{X}_{\tau}\right\},\left\{\mathbf{X}_{\sigma y}\right\}$ from Eq.(21). (8) Given $\left\{\mathbf{X}_{\sigma z}\right\},\left\{\mathbf{X}_{\tau}\right\},\left\{\mathbf{X}_{\sigma y}\right\}$ from Step 7, and the constitutive matrix in Eq. (25b), evaluate the flexibility matrix $[\mathbf{H}]$ from Eq. (32).

(9) Given $\left\{\mathbf{X}_{\sigma z}\right\},\left\{\mathbf{X}_{\tau}\right\},\left\{\mathbf{X}_{\sigma y}\right\}$ from Step 7 and load terms $\sigma_{z}^{*}(y, z), \tau^{*}(y, z), \sigma_{y}^{*}(y, z)$ from Step 6, evaluate the energy conjugate generalized displacement vector $\mathbf{d}_{0}$ from Eq. (33).

(10) Given the flexibility matrix $[\mathbf{H}]$ from Step 8 and $\boldsymbol{\Gamma}(L)$ from Eq. (34), evaluate the element stiffness matrix $[\mathbf{K}]$ from Eq. (42b).

(11) Given the flexibility matrix $[\mathbf{H}]$ from Step 8, $\boldsymbol{\Gamma}(L)$ from Eq. (34), vector $\mathbf{d}_{0}$ from Step 9 and the load terms $\sigma_{z}^{*}(y, z), \tau^{*}(y, z), \sigma_{y}^{*}(y, z)$ from Step 6, evaluate the energy equivalent load vector $\left\{\mathbf{P}_{0}\right\}$ from Eq. (42c).

(12) Use the element stiffness matrix and load vector $[\mathbf{K}]$ and $\left\{\mathbf{P}_{0}\right\}$ obtained in Steps 10 and 11 to form the structure stiffness matrix and load vector and solve for nodal displacements.

(13) Given the nodal displacements, evaluate the augmented nodal vector $\{\mathbf{P}\}$ from Eq. (41) and obtain vector $\{\overline{\mathbf{P}}\}$ from Eq. (42a).

(14) Given $\left\{\mathbf{X}_{\sigma z}\right\},\left\{\mathbf{X}_{\tau}\right\},\left\{\mathbf{X}_{\sigma y}\right\}$ from Step 7, load terms $\sigma_{z}^{*}(y, z), \tau^{*}(y, z), \sigma_{y}^{*}(y, z)$ from Step 6 and $\{\overline{\mathbf{P}}\}$ from Step 13, and setting $z=0, L$, determine the nodal stresses from Eqs. (20).

\section{Appendix C- Flexibility and Stiffness matrices and load vector for Special Case}

A homogeneous beam element with $\operatorname{span} L$ has a rectangular cross-section with dimensions $b \times t$ and is subjected to a uniform traction $\sigma\left(-h_{1}, z\right)=\sigma_{0}$ acting at the top surface while other tractions vanish, i.e., $\tau\left(-h_{1}, z\right)=\sigma\left(h_{2}, z\right)=\tau\left(h_{2}, z\right)=0$. Material is orthotropic with a longitudinal elastic modulus $E_{z}$, transverse elastic modulus $E_{y}$, shear modulus $G$, and Poisson's ratios $\mu_{z y}$ and $\mu_{y z}$ . For the homogeneous beam, the number of layers is set to $p=1$ in Eq. (1). For simplicity, the number of stress terms is taken as $n=3$. The nodal force vector $\{\overline{\mathbf{P}}\}$ in Eq. (17) and the augmented nodal force vector $\{\mathbf{P}\}$ in Eq. (42) are 
$\langle\overline{\mathbf{P}}\rangle_{1 \times 7}^{T}=\left\langle F_{1}(0) \quad F_{1}^{\prime}(0) \quad N(0) \quad Q(0) \quad M(0) \mid F_{1}(L) \quad F_{1}^{\prime}(L)\right\rangle$, and

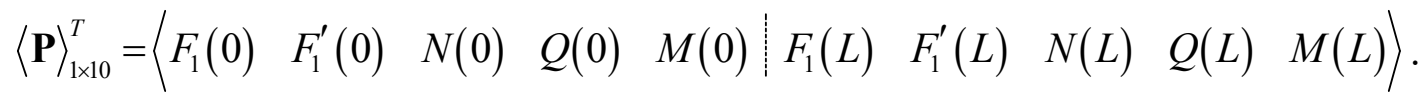

From Eqs. (21), by evaluating $\mathbf{X}_{\sigma z}, \mathbf{X}_{\tau}$, and $\mathbf{X}_{\sigma y}$, and substituting into Eq. (32), the flexibility matrix $[\mathbf{H}]$ is obtained as

$[\mathbf{H}]_{7 \times 7}=\left[\mathbf{H}_{1}\right]+\left[\mathbf{H}_{2}\right]+\left[\mathbf{H}_{3}\right]+\left[\mathbf{H}_{4}\right]$

in which matrix $\left[\mathbf{H}_{1}\right]$ provides the contribution of the longitudinal stresses and is given by

$\left[\mathbf{H}_{1}\right]=\frac{1}{525 E_{z}}\left[\begin{array}{c|c|c|c|c|c|c}156 b t L & & & & & & \\ \hline 22 b t L^{2} & 4 b t L^{3} & & & & s y m s & \\ \hline-210 L & -35 L^{2} & 945 L / b t & & & & \\ \hline 0 & 0 & 0 & 2100 L^{3} / b t^{3} & & & \\ \hdashline 0 & 0 & 0 & 3150 L^{2} / b t^{3} & 6300 L / b t^{3} & & \\ \hdashline-54 b t L & -13 b t L^{2} & 210 L & 0 & 0 & 156 b t L & \\ \hline 13 b t L^{2} & 3 b t L^{3} & -35 L^{2} & 0 & 0 & -22 b t L^{2} & 4 b t L^{3}\end{array}\right]$,

$\left[\mathbf{H}_{2}\right]$ provides the contribution of the coupling terms $\mu_{y z} / E_{z}\left(\right.$ or $\mu_{z y} / E_{y}$ ) and is given by

$\left[\mathbf{H}_{2}\right]=\frac{-\mu_{1 z}}{1575 E_{z}}\left[\begin{array}{c|c|c|c|c|c|c}72 b t^{3} / L & & & & & & \\ \hline 36 b t^{3} & 8 b t^{3} L & & & & s y m s & \\ \hline 0 & -45 t^{2} / 2 & 0 & & & & \\ \hline 0 & 0 & 0 & 0 & & & \\ \hdashline 0 & 0 & 0 & 0 & 0 & & \\ \hline 72 b t^{3} / L & 6 b t^{3} & 0 & 0 & 0 & 72 b t^{3} / L & \\ \hline-6 b t^{3} & 2 b t^{3} L & 45 t^{2} / 2 & 0 & 0 & -36 b t^{3} & 8 b t^{3} L\end{array}\right]$,

$\left[\mathbf{H}_{3}\right]$ provides the contribution of the transverse normal stresses and is given by

$\left[\mathbf{H}_{3}\right]=\frac{b t^{5}}{315 E_{y} L^{3}}\left[\begin{array}{c:c|c|c|c|c|c}6 & & & & & & \\ \hline 3 L & 2 L^{2} & & & & \text { syms } & \\ \hline 0 & 0 & 0 & & & & \\ \hline 0 & 0 & 0 & 0 & & & \\ \hline 0 & 0 & 0 & 0 & 0 & & \\ \hline 6 & 3 L & 0 & 0 & 0 & 6 & \\ \hdashline-3 L & -L^{2} & 0 & 0 & 0 & -3 L & 2 L^{2}\end{array}\right]$

and $\left[\mathbf{H}_{4}\right]$ provides the contribution of the shear stresses and is given by 


$\left[\mathbf{H}_{4}\right]=\frac{1}{525 G}\left[\begin{array}{c|c|c|c|c|c|c}12 b t^{3} / L & & & & & & \\ \hline b t^{3} & 4 b t^{3} L / 3 & & & & s y m s & \\ \hdashline 0 & 0 & 0 & & & & \\ \hline 0 & 0 & 0 & 630 L / b t & & & \\ \hline 0 & 0 & 0 & 0 & 0 & & \\ \hline 12 b t^{3} / L & b t^{3} & 0 & 0 & 0 & 12 b t^{3} / L & \\ \hline-b t^{3} & b t^{3} L / 3 & 0 & 0 & 0 & -b t^{3} & 4 b t^{3} L / 3\end{array}\right]$.

In the present solution, matrices $\left[\mathbf{H}_{1}\right],\left[\mathbf{H}_{2}\right],\left[\mathbf{H}_{3}\right],\left[\mathbf{H}_{4}\right]$ are added and the resulting flexibility matrix $[\mathbf{H}]$ is then inverted. The procedure in Appendix B is then followed to recover the stiffness matrix $[\mathbf{K}]$ and load vector $\left\{\mathbf{P}_{0}\right\}$.

It is of interest to consider the case where matrices $\left[\mathbf{H}_{2}\right],\left[\mathbf{H}_{3}\right],\left[\mathbf{H}_{4}\right]$ are negligible. This corresponds to omitting the shear and transverse normal stresses and orthotropic coupling effects. In this case, the resulting stiffness matrix takes the form:

$[\mathbf{K}]=$

\begin{tabular}{|c|c|c|c|c|c|c|c|c|c|}
\hline \multicolumn{10}{|l|}{$21 E / A L$} \\
\hline$-150 E / A L^{2}$ & $1500 E / A L^{3}$ & & & & & & & & \\
\hline$E / L$ & 0 & $E A / L$ & & & & & syms & & \\
\hline 0 & 0 & 0 & $12 E I / L^{3}$ & & & & & & \\
\hline 0 & 0 & 0 & $-6 E I / L^{2}$ & $4 E I / L$ & & & & & \\
\hline $4 E / A \bar{L}$ & $-75 E / A L^{2-}$ & $-E / L$ & 0 & 0 & $21 E / A L$ & $150 E A / L^{2}$ & & & \\
\hline $75 E / A L^{2}$ & $-1050 E / A L^{3}$ & 0 & 0 & 0 & $150 E / A L^{2}$ & $1500 E A / L^{3}$ & & & \\
\hline$-E / L$ & 0 & $-E A / L$ & 0 & 0 & $E / L$ & 0 & $E A / L$ & & \\
\hline 0 & 0 & 0 & $-12 E I / L^{3}$ & $6 E I / L^{3}$ & 0 & 0 & 0 & $12 E I / L^{3}$ & \\
\hline 0 & 0 & 0 & $-6 E I / L^{3}$ & $2 E I / L$ & 0 & 0 & 0 & $6 E I / L^{2}$ & $4 E I / L$ \\
\hline
\end{tabular}

in which the terms $A=b t, I=b t^{3} / 12$ and $E=E_{z}$ have been introduced. It is of interest to note that the boxed entries match those of the classical Euler Bernoulli beam element. Also, the nodal force vector is found to take the form

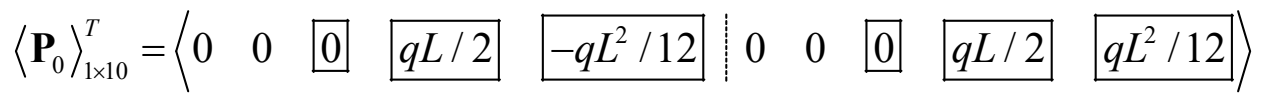

with $q=\sigma_{0} b$. Again, for the boxed terms correspond to the energy equivalent load vector for an Euler Bernoulli beam element subjected to uniformly distributed load. 


\section{References}

[1] Timoshenko, S. P. On the corrections for shear of the differential equation for transverse vibrations of prismatic bars, Philosophical magazine, 41 (1921) 744-746.

[2] Cowper, G. R., The Shear Coefficient in Timoshenko's Beam Theory, Journal of applied mechanics, 33 (1966) 335-340.

[3] Stephen, N. G., Timoshenko's shear coefficient from a beam subjected to gravity loading, Journal of Applied Mechanics, 47 (1980) 121-127.

[4] Kaneko, T., On Timoshenko's correction for shear in vibrating beams, Journal of physics D: Applied Physics, 8 (1975) 1927-1936.

[5] Blevins, R., D., Formulas for Dynamics, Acoustics and Vibration, Wiley Publisher, ISBN: 978-1-119-03811-5, 2015.

[6] Timoshenko, S. P., On the transverse vibrations of bars of uniform cross-section, Philosophical magazine, 125, 1922.

[7] Mindlin, R. D., Deresiewicz, H., Timoshenko's Shear Coefficient for Flexural Vibrations of Beams, Technical Report No. 10, ONR Project NR064-388, Department of Civil Engineering, Columbia University, New York, N. Y, 1953.

[8] Hutchinson, J. R., Transverse vibration of beams, exact versus approximate solutions, Journal of applied mechanics. 48 (1981) 923-928.

[9] Heyliger, P. R. and Reddy, J. N., A higher order beam finite element for bending and vibration problems, Journal of sound and vibration.126 (1988) 309-326.

[10] Stephen, N. G. and Levinson, M., A second order beam theory, Journal of Sound and Vibration. 67 (1979) 293-305.

[11] Reddy, J., Energy and variational methods in applied mechanics, John Wiley \& Sons, 1984.

[12] Shu, X., and Sun, L., An improved simply higher order theory for laminated composite plates, Computers \&Structures. 50 (1994) 231-236.

[13] Jha, D., K., Kant, T., and Singh, R., K., Stress analysis of transversely loaded functionally graded plates with a higher order shear and normal deformation theory, Journal of engineering mechanics. 139 (2013), 1663-1680.

[14] Kant, T. and Manjunatha, B.S., Higher-order theories for symmetric and unsymmetric fiber reinforced composite beams with C0 finite elements, Finite Ele. Ana. \& Des., 6 (1990) 303-320. [15] Zhang, S., Yin, J., Zhang, H.W., and Chen, B.S., A two-level method for static and dynamic analysis of multilayered composite beam and plate, Finite Ele. Ana. \& Des., 111 (2016) 1-18. 
[16] Wu, X., F., and Jensen, R. A., Stress function variational method for stress analysis of bonded joints under mechanical and thermal loads, Int. J. of engineering science. 49 (2011) 279-294.

[17] Wu, X., F., and Jensen, R. A., Semianalytic stress-function variational approach for the interfacial stresses in bonded joints, Journal of engineering mechanics. 140 (2014) 1-11.

[18] Carrera, E., and Guinta, G., Refined beam theories based on a unified formulation, International journal of applied mechanics, 2 (2010) 117-143.

[19] Carrera, E., Pagani, A., and Zangallo, F., Comparison of various 1D, 2D, and 3D models for the analysis of thin-walled box with transverse ribs subjected to load factors, Journal of finite elements in analysis and design, 95 (2015) 1-11.

[20] Groh, R. M. J., and Weaver, P. M., Static inconsistencies in certain axiomatic higher-order shear deformation theories for beams, plates and shells, Composite Structs., 120 (2015) 231-245. [21] Groh, R.M., J. and Tessler, A., (2017), Computationally efficient beam elements for accurate stresses in sandwich laminates and laminated composites with delamination, Computer Methods in Applied Mechanics and Engineering, 320 (1), 369-395.

[22] Wunderlich, W., and Pilkey, W., D., Mechanics of Structures: Variational and Computational Methods, second edition, CRC Press LLC, Frorida 33431, 2003.

[23] Erkmen, R., E., and Mohareb, M., Torsion analysis of thin-walled beams including shear deformation effects, Journal of thin walled structures, 46 (2008) 1096-1108.

[24] Erkmen, R., E., and Mohareb, M., Buckling analysis of thin-walled open members- A complementary energy variational principle, Journal of thin walled structures, 46 (2008) 602-617. [25] Zhao, B., Lu, Z.H., and Lu, Y., N., Two-dimensional analytical solution of elastic stresses for balanced single-lap joints-Variational method, J. adhesion and Adhesives, 49 (2014) 115-126. [26] Timoshenko, S.P. and Goodier, J.,N., Theory of elasticity (McGwaw-Hill, New York), 1970. [27] Pham, P.V. and Mohareb, M., A shear deformable theory for the analysis of steel beams reinforced with GFRP plates, Thin-walled structures, 85 (2014) 165-182.

[28] Pham, P.V. and Mohareb, M., Finite-Element Formulations for the Spatial Static Response of Steel Beams Bonded to a GFRP Plate, J. of engineering mechanics, 141 (2015) 04014143. [29] Hexcel Corporation, HexWeb TM Honeycomb Sandwich Design Technology, website: http://www.hexcel.com/Resources/DataSheets/Brochure Data Sheets/ Honeycomb Sandwich Design Technology.pdf, (2000). 
[30] Pappada, S., Rametta, R., and Lanzilotto, L., Effect of manufacturing process and skin-core adhesion efficiency on the mechanical properties of a thermoplastic sandwich. In: ICCM 17, Edinburgh, Scottland (2009) 27-31.

[31] Sarangan, S. and Singh, B.N., Improved zigzag theories for laminated composite and sandwich plates with interlaminar shear stress continuity, Aerospace Science and Technology, 52 (2016) 243-255. 